\title{
Adducts of Tris(alkyl) Holmium(III) Showing Magnetic Relaxation
}

Shi-Ming Chen, † Yi-Quan Zhang, † Jin Xiong, + Bing-Wu Wang*, , Song Gao*+

† Beijing National Laboratory of Molecular Science, State Key Laboratory of Rare Earth Materials Chemistry and Applications, College of Chemistry and Molecular Engineering, Peking University, Beijing 100871, P. R. China.

‡ Jiangsu Key Laboratory for NSLSCS, School of Physical Science and Technology, Nanjing Normal University, Nanjing 210023, P. R. China. 


\section{Table of Contents}

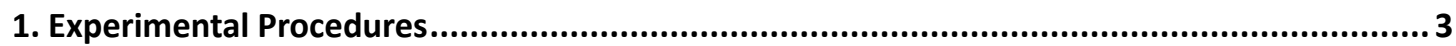

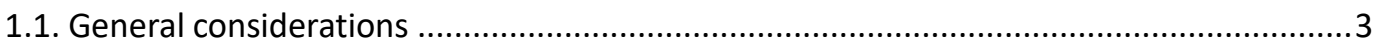

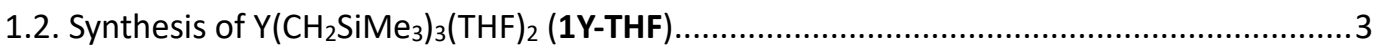

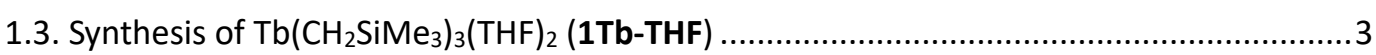

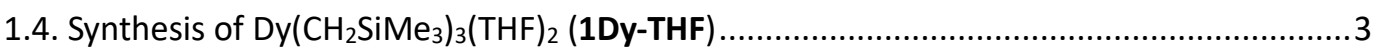

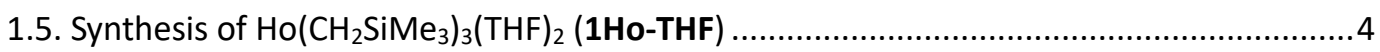

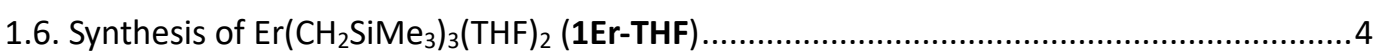

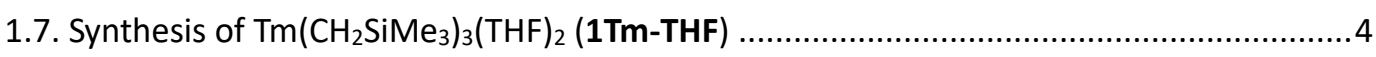

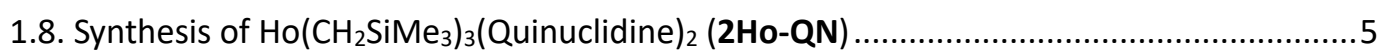

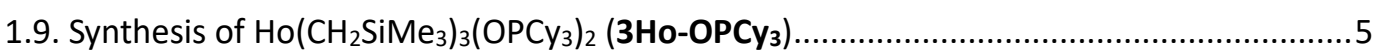

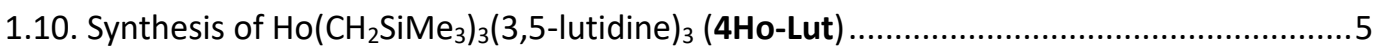

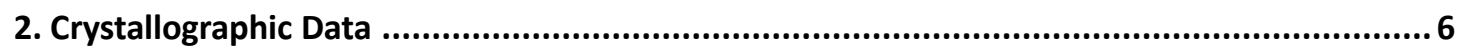

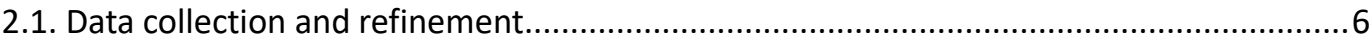

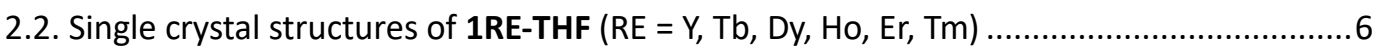

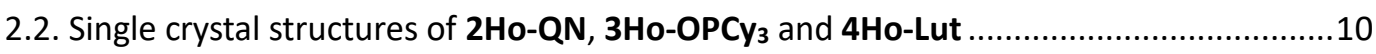

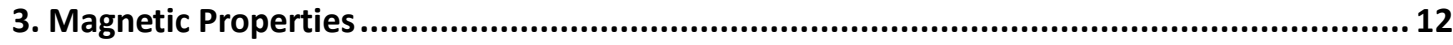

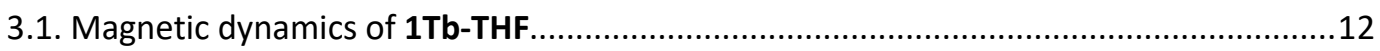

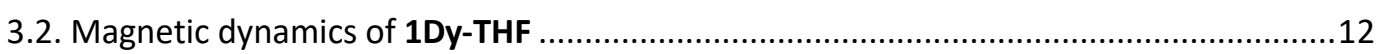

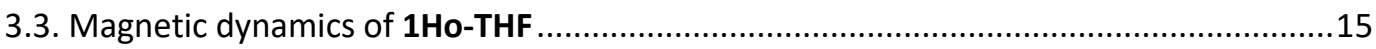

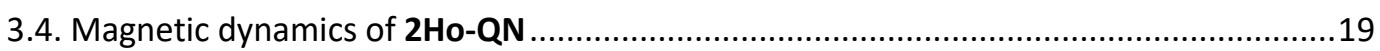

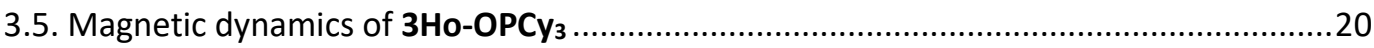

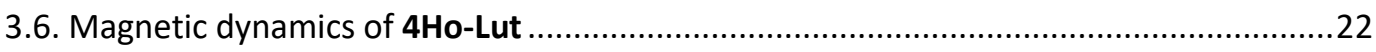

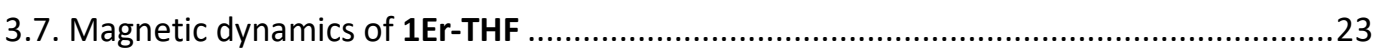

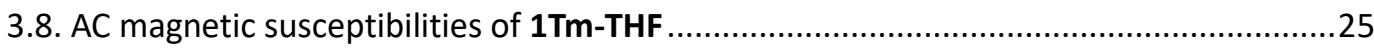

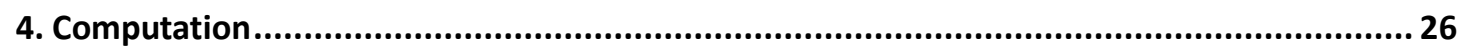

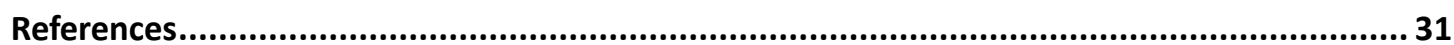




\section{Experimental Procedures}

\subsection{General considerations}

All reactions and manipulations described below were carried out under anhydrous and anaerobic conditions using Ar-filled glovebox. Tetrahydrofuran (THF) and $n$-hexane were degassed and dehydrated by solvent purification system. Anhydrous $\mathrm{YCl}_{3}, \mathrm{TbCl}_{3}, \mathrm{DyCl}_{3}, \mathrm{HoCl}_{3}, \mathrm{ErCl}_{3}, \mathrm{TmCl}_{3}$, quinuclidine (QN) and $\mathrm{OPCу}_{3}$ are commercially available and directly used without further purification. $\mathrm{LiCH}_{2} \mathrm{SiMe}_{3}(0.55 \mathrm{~mol} / \mathrm{L}$ in $n$-hexane) was purchased from J\&K and used as white powder after the solvent being removed under vacuum. 3,5-dimethylpyridine (lutidine) was dried, and then distilled under reduced pressure before use. Elemental analyses were performed on the Vario EL CUBE Elemental Analyzer with the sample tightly wrapped by three layers of tin foil. The syntheses of the heavy lanthanide series of $\mathbf{1} \mathbf{L n}$-THF were according to the previous literature with major modifications ${ }^{1}$.

\subsection{Synthesis of $\mathrm{Y}\left(\mathrm{CH}_{2} \mathrm{SiMe}_{3}\right)_{3}(\mathrm{THF})_{2}$ (1Y-THF)}

In an Ar-filled glovebox, $0.9763 \mathrm{~g}(5 \mathrm{mmol})$ of anhydrous $\mathrm{YCl}_{3}$ were suspended and vigorously stirred in THF. $1.4124 \mathrm{~g}$ (15 mmol) of $\mathrm{LiCH}_{2} \mathrm{SiMe}_{3}$ powder were dissolved in $5 \mathrm{~mL}$ of THF and added dropwise to the above $\mathrm{YCl}_{3}$ suspension. The reaction solution was stirred for $40 \mathrm{~min}$. After that solvent was removed under vacuum and the remaining grey slurry was extracted with hexane ( $7 \times 10$ $\mathrm{mL}$ ). The hexane extracts were combined and filtered through a glass frit funnel to remove the insoluble $\mathrm{LiCl}$ salt. The filtrate was concentrated and cooled in -30 oC overnight. Then the solution was transferred to a $25 \mathrm{~mL}$ of flask and the yellow oil at the bottom was discarded if any. The solution was further concentrated under vacuum and then cooled in -30 o C. After several days, light yellow single crystals suitable for X-ray diffraction were obtained in good yields (1.8086 $\mathrm{g}$, 73\%). Elemental analysis for 1Y-THF: C\%, 48.86 (48.55); H\%, 9.91 (9.98) and the numbers in the parenthesis is theoretical values.

\subsection{Synthesis of $\mathrm{Tb}\left(\mathrm{CH}_{2} \mathrm{SiMe}_{3}\right)_{3}(\mathrm{THF})_{2}$ (1Tb-THF)}

In an Ar-filled glovebox, $1.3264 \mathrm{~g}$ ( $5 \mathrm{mmol}$ ) of anhydrous $\mathrm{TbCl}_{3}$ were suspended and vigorously stirred in THF. $1.4124 \mathrm{~g} \mathrm{(15} \mathrm{mmol)} \mathrm{of} \mathrm{LiCH}_{2} \mathrm{SiMe}_{3}$ powder were dissolved in $5 \mathrm{~mL}$ of THF and added dropwise to the above $\mathrm{TbCl}_{3}$ suspension. The reaction solution was stirred for $40 \mathrm{~min}$. After that solvent was removed under vacuum and the remaining pale yellow/grey slurry was extracted with hexane $(7 \times 10 \mathrm{~mL})$. The hexane extracts were combined and filtered through a glass frit funnel to remove the insoluble $\mathrm{LiCl}$ salt. The filtrate was concentrated and cooled in -30 o $\mathrm{C}$ overnight. Then the solution was transferred to a $25 \mathrm{~mL}$ of flask and the dark yellow oil at the bottom was discarded if any. The solution was further concentrated under vacuum and then cooled in -30 oc . After several days, yellow single crystals suitable for X-ray diffraction were obtained in good yields (1.9297 g, 68\%). Elemental analysis for 1Tb-THF: C\%, 42.28 (42.53); H\%, 8.65 (8.75).

\subsection{Synthesis of $\mathrm{Dy}\left(\mathrm{CH}_{2} \mathrm{SiMe}_{3}\right)_{3}(\mathrm{THF})_{2}$ (1Dy-THF)}

In an Ar-filled glovebox, $1.3443 \mathrm{~g}(5 \mathrm{mmol})$ of anhydrous $\mathrm{DyCl}_{3}$ were suspended and vigorously 
stirred in THF. $1.4124 \mathrm{~g}$ (15 mmol) of $\mathrm{LiCH}_{2} \mathrm{SiMe}_{3}$ powder were dissolved in $5 \mathrm{~mL}$ of THF and added dropwise to the above $\mathrm{DyCl}_{3}$ suspension. The reaction solution was stirred for $40 \mathrm{~min}$. After that solvent was removed under vacuum and the remaining pale grey slurry was extracted with hexane $(7 \times 10 \mathrm{~mL})$. The hexane extracts were combined and filtered through a glass frit funnel to remove the insoluble LiCl salt. The filtrate was concentrated and cooled in $-30 \stackrel{\circ}{\circ}$ overnight. Then the solution was transferred to a $25 \mathrm{~mL}$ of flask and the dark yellow oil at the bottom was discarded if

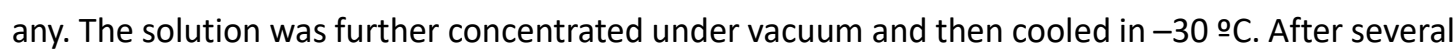
days, light yellow single crystals suitable for X-ray diffraction were obtained in good yields (2.0257 g, 71\%). Elemental analysis for 1Dy-THF: C\%, 41.89 (42.27); H\%, 8.78 (8.69).

\subsection{Synthesis of $\mathrm{Ho}\left(\mathrm{CH}_{2} \mathrm{SiMe}_{3}\right)_{3}(\mathrm{THF})_{2}(1 \mathrm{Ho}-\mathrm{THF})$}

In an Ar-filled glovebox, $1.3564 \mathrm{~g}(5 \mathrm{mmol})$ of anhydrous $\mathrm{HoCl}_{3}$ were suspended and vigorously stirred in THF. $1.4124 \mathrm{~g}(15 \mathrm{mmol})$ of $\mathrm{LiCH}_{2} \mathrm{SiMe}_{3}$ powder were dissolved in $5 \mathrm{~mL}$ of THF and added dropwise to the above $\mathrm{HoCl}_{3}$ suspension. The reaction solution was stirred for $40 \mathrm{~min}$ and gradually turned clear. After that solvent was removed under vacuum and the remaining grey slurry was extracted with hexane $(7 \times 10 \mathrm{~mL})$. The hexane extracts were combined and filtered through a glass

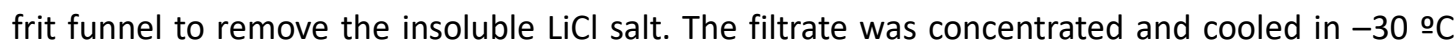
overnight. Then the solution was transferred to a $25 \mathrm{~mL}$ of flask and the yellow oil at the bottom was discarded if any. The solution was further concentrated under vacuum and then cooled in -30 ㅇ. After several days, orange yellow single crystals suitable for X-ray diffraction were obtained in good yields (2.0196 g, 71\%). Elemental analysis for 1Ho-THF: C\%, 41.74 (42.09); H\%, 8.61 (8.65).

\subsection{Synthesis of $\operatorname{Er}\left(\mathrm{CH}_{2} \mathrm{SiMe}_{3}\right)_{3}(\mathrm{THF})_{2}$ (1Er-THF)}

In an Ar-filled glovebox, $1.3681 \mathrm{~g} \mathrm{(5} \mathrm{mmol)} \mathrm{of} \mathrm{anhydrous} \mathrm{ErCl}_{3}$ were suspended and vigorously stirred in THF. $1.4124 \mathrm{~g}(15 \mathrm{mmol})$ of $\mathrm{LiCH}_{2} \mathrm{SiMe}_{3}$ powder were dissolved in $5 \mathrm{~mL}$ of THF and added dropwise to the above $\mathrm{ErCl}_{3}$ suspension. The reaction solution was stirred for $40 \mathrm{~min}$. After that solvent was removed under vacuum and the remaining dark grey slurry was extracted with hexane $(7 \times 10 \mathrm{~mL})$. The hexane extracts were combined and filtered through a glass frit funnel to remove the insoluble LiCl salt. The filtrate was concentrated and cooled in $-30 \stackrel{\circ}{\circ}$ overnight. Then the solution was transferred to a $25 \mathrm{~mL}$ of flask and the dark orange oil at the bottom was discarded if any. The solution was further concentrated under vacuum and then cooled in $-30 \stackrel{\circ}{ } \mathrm{C}$. After several days, light pink single crystals suitable for X-ray diffraction were obtained in good yields $(2.1011 \mathrm{~g}$, 73\%). Elemental analysis for 1Er-THF: C\%, 41.62 (41.91); H\%, 8.46 (8.62).

\subsection{Synthesis of $\mathrm{Tm}\left(\mathrm{CH}_{2} \mathrm{SiMe}_{3}\right)_{3}(\mathrm{THF})_{2}(1 \mathrm{Tm}$-THF)}

In an Ar-filled glovebox, $1.3764 \mathrm{~g} \mathrm{(5} \mathrm{mmol)} \mathrm{of} \mathrm{anhydrous} \mathrm{TmCl}_{3}$ were suspended and vigorously stirred in THF. $1.4124 \mathrm{~g}(15 \mathrm{mmol})$ of $\mathrm{LiCH}_{2} \mathrm{SiMe}_{3}$ powder were dissolved in $5 \mathrm{~mL}$ of THF and added dropwise to the above $\mathrm{TmCl}_{3}$ suspension. The reaction solution was stirred for $40 \mathrm{~min}$. After that solvent was removed under vacuum and the remaining light grey slurry was extracted with hexane $(7 \times 10 \mathrm{~mL})$. The hexane extracts were combined and filtered through a glass frit funnel to remove the insoluble $\mathrm{LiCl}$ salt. The filtrate was concentrated and cooled in $-30 \stackrel{\circ}{\circ}$ overnight. Then the solution was transferred to a $25 \mathrm{~mL}$ of flask and the yellow oil at the bottom was discarded if any. 
The solution was further concentrated under vacuum and then cooled in $-30 \stackrel{\circ}{ }$. After several days, light yellow single crystals suitable for X-ray diffraction were obtained in good yields $(2.0055 \mathrm{~g}$, 70\%). Elemental analysis for 1Tm-THF: C\%, 41.54 (41.79); H\%, 8.41 (8.59).

\subsection{Synthesis of $\mathrm{Ho}\left(\mathrm{CH}_{2} \mathrm{SiMe}_{3}\right)_{3}$ (Quinuclidine $)_{2}(2 \mathrm{Ho}-\mathrm{QN})$}

To a toluene solution of 1 Ho-THF $(0.2854 \mathrm{~g}, 0.5 \mathrm{mmol})$ were added $0.1112 \mathrm{~g}(1.0 \mathrm{mmol})$ of quinuclidine at room temperature. The reaction mixture was stirred for $40 \mathrm{~min}$ followed by concentration under vacuum. The concentrated solution was cooled in $-30 \circ \mathrm{C}$ for several days and yellow single crystals suitable for X-ray diffraction were obtained in good yields $(0.2835 \mathrm{~g}, 87 \%)$. Elemental analysis for 2Ho-QN: C\%, 48.18(48.12); H\%, 9.23(9.16); N\%, 3.97(4.32).

\subsection{Synthesis of $\mathrm{Ho}\left(\mathrm{CH}_{2} \mathrm{SiMe}_{3}\right)_{3}\left(\mathrm{OPC}_{3}\right)_{2}\left(3 \mathrm{Ho}-\mathrm{OPC} y_{3}\right)$}

In an analogous way to $2 \mathrm{Ho}-\mathrm{QN}$, to a $1 \mathrm{~mL}$ toluene solution of $1 \mathrm{Ho}-\mathrm{THF}(0.2854 \mathrm{~g}, 0.5 \mathrm{mmol})$ were added $5 \mathrm{~mL}$ of toluene solution of $\mathrm{OPC}_{3}(0.2964 \mathrm{~g}, 1.0 \mathrm{mmol})$ at room temperature. The reaction mixture was stirred for $3 \mathrm{~h}$ followed by concentration under vacuum. The concentrated solution was cooled in $-30{ }^{\circ} \mathrm{C}$ for one day and yellow single crystals suitable for $\mathrm{X}$-ray diffraction were obtained in good yields $(0.4258 \mathrm{~g}, 84 \%)$. Elemental analysis for $3 \mathrm{Ho}^{-O} \mathrm{OPC}_{3}: \mathrm{C} \%, 56.91(56.55)$; $\mathrm{H} \%$, 9.93(9.79).

\subsection{Synthesis of $\mathrm{Ho}\left(\mathrm{CH}_{2} \mathrm{SiMe}_{3}\right)_{3}(3,5 \text {-lutidine })_{3}$ (4Ho-Lut)}

$0.8138 \mathrm{~g}$ ( $3 \mathrm{mmol}$ ) of $\mathrm{HoCl}_{3}$ were solvated in THF. THF solution of $\mathrm{LiCH}_{2} \mathrm{SiMe}_{3}(0.8474 \mathrm{~g}, 9 \mathrm{mmol})$ was added dropwise to the above suspension. After that, 3,5-lutidine $(0.6430 \mathrm{~g}, 6 \mathrm{mmol})$ were added slowly to the above solution. The reaction mixture was stirred for $40 \mathrm{~min}$ and then solvent was removed under vacuum. Then the yellow grey slurry was extracted by hexane $(7 \times 10 \mathrm{~mL})$. The hexane extracts were combined and filtered through a glass frit funnel to remove the insoluble LiCl salt. The filtrate was concentrated and cooled in -30 으 overnight. Then the solution was transferred to a $25 \mathrm{~mL}$ of flask and the yellow oil at the bottom was discarded if any. The solution was further concentrated under vacuum and then cooled in -30 ㄷ. After two days, orange yellow single crystals suitable for $X$-ray diffraction were obtained in good yields $(0.2324 \mathrm{~g}, 62 \%)$. Elemental analysis for 4Ho-Lut: C\%, 53.04(52.99); H\%, 8.02(8.09); N\%, 5.46(5.62). 


\section{Crystallographic Data}

\subsection{Data collection and refinement}

The crystals were wrapped in mineral oil and then were frozen in $180 \mathrm{~K}$. Data collections were performed at $180 \mathrm{~K}$ on a SuperNova diffractometer using graphite-monochromated Mo $\mathrm{K} \alpha$ radiation $(\lambda=0.71073 \AA)$. Using Olex $2^{[2]}$, the structure was solved with the Superflip structure solution program using Charge Flipping ${ }^{[3]}$ and refined with the ShelXL refinement package using Least Squares minimization. Refinement was performed on $F^{2}$ anisotropically for all of the nonhydrogen atoms by the full-matrix least-squares method. The hydrogen atoms were placed at the calculated positions and were included in the structure calculation without further refinement of their parameters. Crystallographic data (excluding structure factors) have been deposited with the Cambridge Crystallographic Data Centre with supplementary publication numbers: 1909166 for 1Y-THF, 1909168 for 1Tb-THF, 1909165 for 1Dy-THF, 1909169 for 1Ho-THF, 1909167 for 1ErTHF, 1909164 for 1Tm-THF, 1950992 for 2Ho-QN, 1950994 for 3Ho-OPCy 3 and 1950993 for 4HoLut. These data can be obtained free of charge from the Cambridge Crystallographic Data Centre via https://www.ccdc.cam.ac.uk/structures/.

\subsection{Single crystal structures of 1 RE-THF (RE = Y, Tb, Dy, Ho, Er, Tm)}

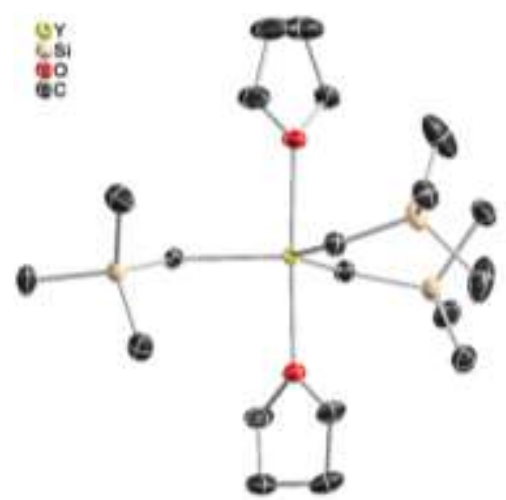

(a)

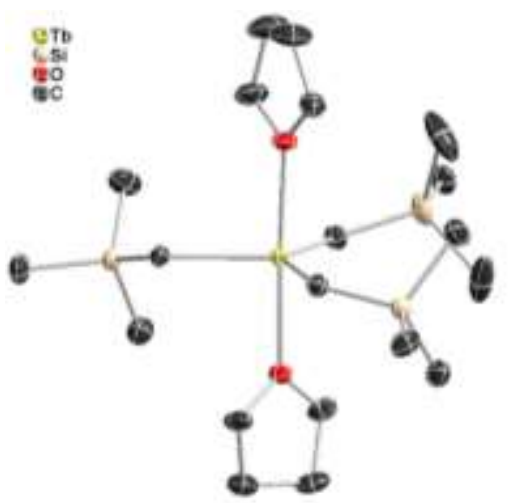

(c)

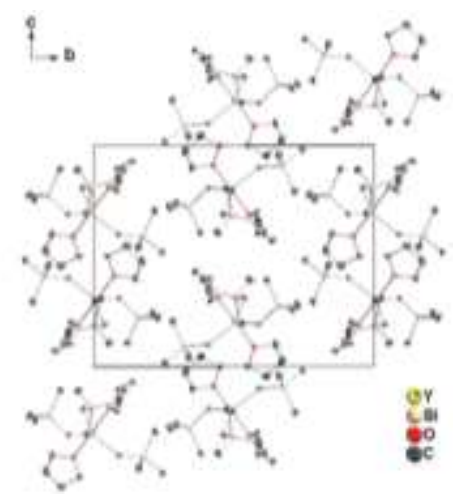

(b)

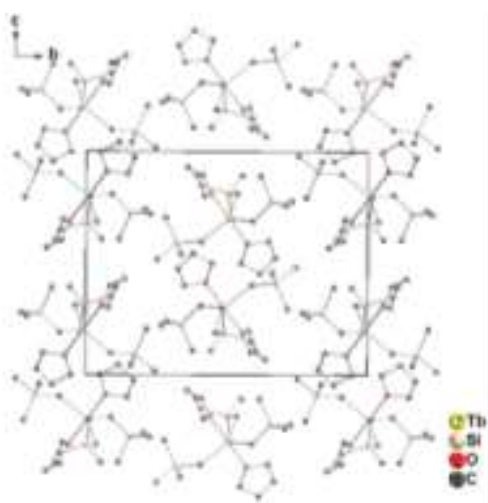

(d) 


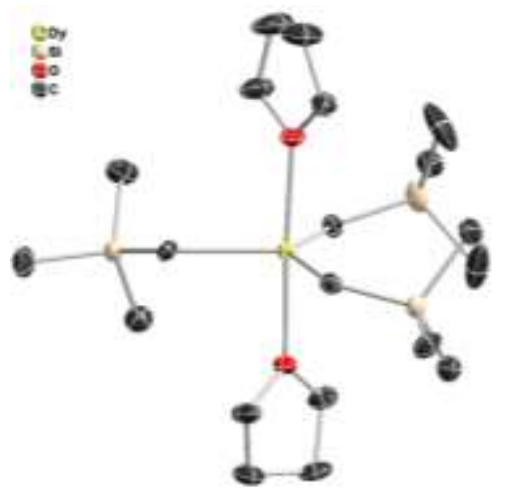

(e)

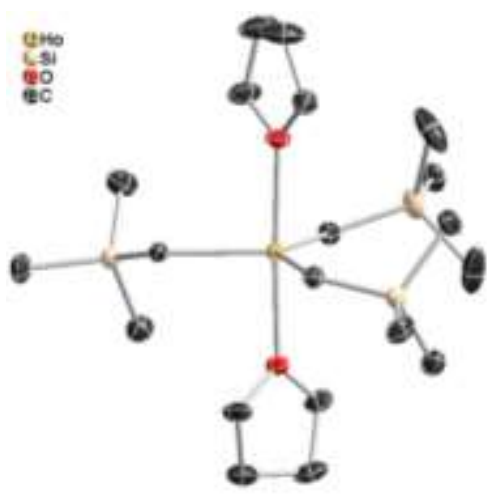

(g)

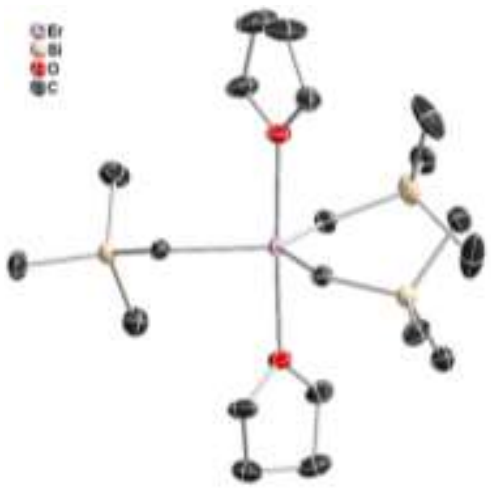

(i)

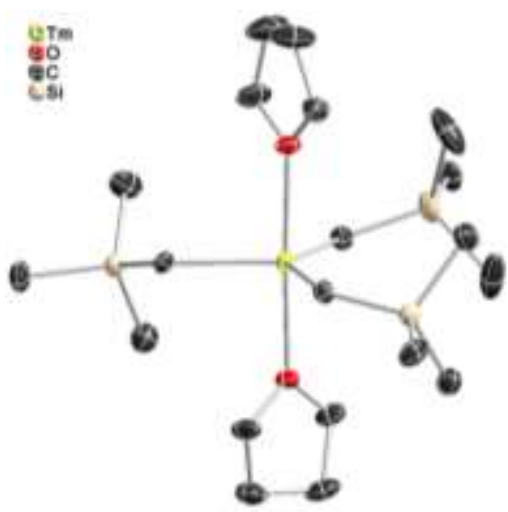

(k)

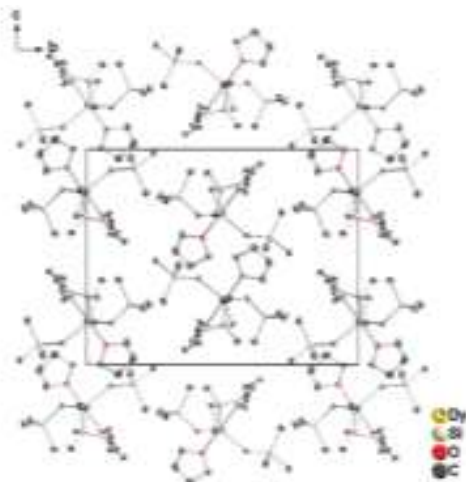

(f)

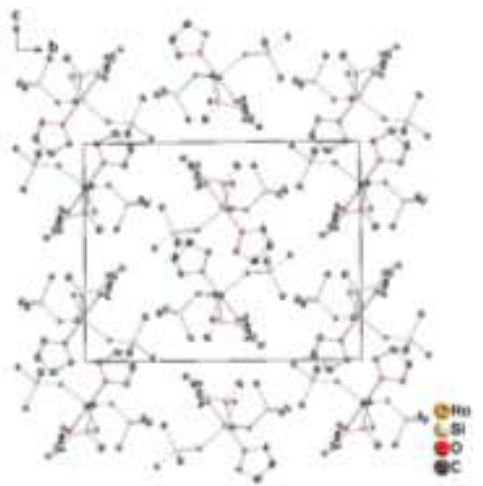

(h)

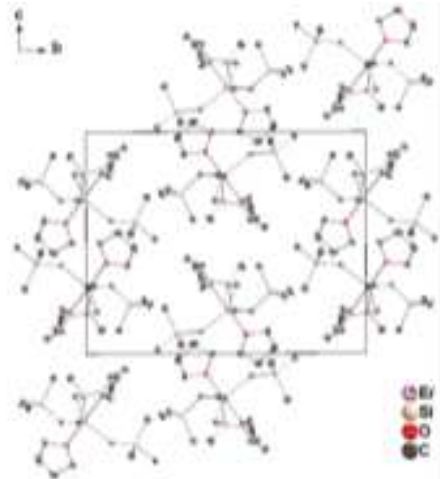

(i)

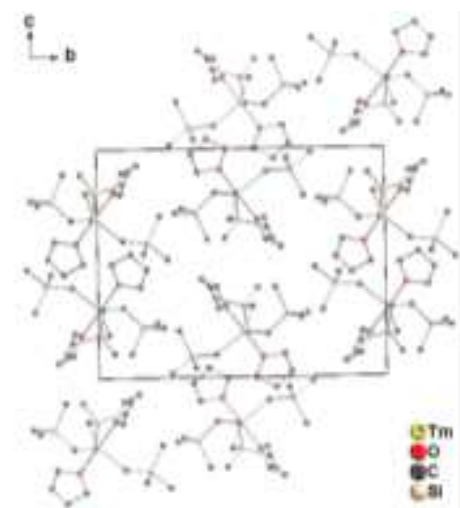

(I)

Figure S1. Molecular structure (a, c, e, g, i, k) and packing diagram (b, d, f, h, j, I) of 1Y-THF, 1Tb-THF, 1Dy-THF, 1HoTHF, 1Er-THF, 1Tm-THF, respectively. Hydrogen atoms have been omitted for clarity. 
Table S1. Crystallographic data of $\mathbf{1} \mathrm{Ln}-\mathrm{THF}$ ( $\mathrm{Ln}=\mathrm{Tb}$, Dy, Ho, Er, Tm)

\begin{tabular}{|c|c|c|c|c|c|}
\hline Formula & $\mathrm{C}_{20} \mathrm{H}_{40} \mathrm{O}_{2} \mathrm{Si}_{3} \mathrm{~Tb}$ & $\mathrm{C}_{20} \mathrm{H}_{40} \mathrm{O}_{2} \mathrm{Si}_{3} \mathrm{Dy}$ & $\mathrm{C}_{20} \mathrm{H}_{49} \mathrm{O}_{2} \mathrm{Si} 3 \mathrm{Ho}$ & $\mathrm{C}_{20} \mathrm{H}_{40} \mathrm{O}_{2} \mathrm{Si}_{3} \mathrm{Er}$ & $\mathrm{C}_{20} \mathrm{H}_{40} \mathrm{O}_{2} \mathrm{Si}, \mathrm{Tm}$ \\
\hline Formula Weight & 564.78 & 568.36 & 570.79 & 573.12 & 574.79 \\
\hline$T, K$ & $180(2)$ & $180(2)$ & $180(2)$ & $180(2)$ & $180(2)$ \\
\hline$\lambda, A$ & 0.71073 & 0.71073 & 0.71073 & 0.71073 & 0.71073 \\
\hline Crystal System & Monoclinic & Monoclinic & Monoclinic & Monoclinic & Monoclinic \\
\hline Space Group & $P_{2} / n$ & $P 2_{1} / n$ & $P 2, / n$ & $P_{2} / n$ & $P_{21} / n$ \\
\hline$a, A$ & $10.6830(2)$ & $10.6955(4)$ & $10.6774(2)$ & $10.6732(3)$ & $10.6679(3)$ \\
\hline$b, A$ & $18.5962(4)$ & $18.5910(6)$ & $18.6057(4)$ & $18.5924(5)$ & $18.5675(5)$ \\
\hline$c, A$ & $14.8024(3)$ & $14.8322(7)$ & $14.7623(3)$ & $14.7455(4)$ & $14.7483(4)$ \\
\hline 6, & $95.110(2)$ & $95.100(3)$ & $95.2816(18)$ & $95.360(3)$ & $95.273(3)$ \\
\hline$z$ & 4 & 4 & 4 & 4 & 4 \\
\hline$V, A^{\prime}$ & $2928.98(11)$ & $2937.56(19)$ & $2920.23(10)$ & $2913.31(14)$ & $2908.92(14)$ \\
\hline$D_{e}, \mathrm{~g} \mathrm{~cm}^{-3}$ & 1.281 & 1.285 & 1.298 & 1.307 & 1.312 \\
\hline$F(000)$ & 1168.0 & 1172.0 & 1176.0 & 1180.0 & 1184.0 \\
\hline$\mu_{1} \mathrm{~mm}^{-4}$ & 2.548 & 2.676 & 2.843 & 3.021 & 3.184 \\
\hline$R_{1}$ (reflections) & $0.0337(6291)$ & $0.0405(5321)$ & $0.0312(6373)$ & $0.0322(5347)$ & $0.0318(5543)$ \\
\hline$w R_{2}$ (reflections) & $0.0684(8325)$ & $0.0828(7518)$ & $0.0605(8244)$ & $0.0650(6923)$ & $0.0628(7201)$ \\
\hline$s$ & 1.077 & 1.052 & 1.075 & 1.089 & 1.074 \\
\hline
\end{tabular}


Table S2. Selected bond lengths and angles of $\mathbf{1} \mathrm{Ln}-\mathrm{THF}$ ( $\mathrm{Ln}=\mathrm{Tb}$, Dy, Ho, Er, Tm)

\begin{tabular}{|c|c|c|c|c|c|}
\hline Bond/Angle & $\mathrm{C}_{20} \mathrm{H}_{49} \mathrm{O}_{2} \mathrm{Si}_{3} \mathrm{~Tb}$ & $\mathrm{C}_{20} \mathrm{H}_{49} \mathrm{O}_{2} \mathrm{Si}_{3} \mathrm{Dy}$ & $\mathrm{C}_{20} \mathrm{H}_{49} \mathrm{O}_{2} \mathrm{Si}_{3} \mathrm{Ho}$ & $\mathrm{C}_{20} \mathrm{H}_{49} \mathrm{O}_{2} \mathrm{Si}_{3} \mathrm{Er}$ & $\mathrm{C}_{20} \mathrm{H}_{49} \mathrm{O}_{2} \mathrm{Si}_{3} \mathrm{Tm}$ \\
\hline Ln-01, A & $2.391(2)$ & $2.384(3)$ & $2.362(2)$ & $2.349(2)$ & $2.342(2)$ \\
\hline $\operatorname{Ln}-02, A$ & $2.378(2)$ & $2.375(3)$ & $2.352(2)$ & $2.338(2)$ & $2.332(2)$ \\
\hline $\operatorname{Ln}-\mathrm{C}_{1}, \mathrm{~A}$ & $2.416(3)$ & $2.406(4)$ & $2.399(3)$ & $2.393(4)$ & $2.372(3)$ \\
\hline $\operatorname{Ln}-\mathrm{C} 2, A$ & $2.407(3)$ & $2.403(4)$ & $2.389(3)$ & $2.374(3)$ & $2.362(3)$ \\
\hline $\operatorname{Ln}-\mathrm{C} 3, \mathrm{~A}$ & $2.435(3)$ & $2.427(5)$ & $2.412(3)$ & $2.406(4)$ & $2.389(3)$ \\
\hline Si1-C1, $\AA$ & $1.831(3)$ & $1.837(4)$ & $1.836(3)$ & $1.831(4)$ & $1.837(3)$ \\
\hline $\mathrm{Si} 2-\mathrm{C} 2, \AA$ & $1.827(3)$ & $1.834(4)$ & $1.830(3)$ & $1.826(4)$ & $1.831(4)$ \\
\hline $\mathrm{Si} 3-\mathrm{C} 3, \AA$ & $1.829(3)$ & $1.835(4)$ & $1.831(3)$ & $1.829(4)$ & $1.832(3)$ \\
\hline O1-Ln-02, " & $177.55(8)$ & $177.26(10)$ & $177.79(7)$ & $177.75(9)$ & $177.48(9)$ \\
\hline C1-Ln-C2, * & $109.53(11)$ & $109.03(15)$ & $109.99(10)$ & $110.22(12)$ & $109.96(11)$ \\
\hline C1- $\ln -\mathrm{C} 3$, & $134.44(11)$ & $135.12(14)$ & $134.00(11)$ & $133.79(13)$ & $134.01(12)$ \\
\hline $\mathrm{C} 2-\operatorname{Ln}-\mathrm{C}_{3}$ & $115.89(11)$ & $115.70(14)$ & $115.89(10)$ & $115.88(13)$ & $115.94(12)$ \\
\hline Ln-C1-Si1, & $129.15(16)$ & $129.0(2)$ & $129.57(16)$ & $129.7(2)$ & $129.79(17)$ \\
\hline $\mathrm{Ln}-\mathrm{C} 2-\mathrm{Si} 2$ & $123.05(15)$ & $122.9(2)$ & $123.19(14)$ & $123.55(19)$ & $123.63(16)$ \\
\hline Ln-C3-Si3, " & $127.08(16)$ & $126.8(2)$ & $127.39(16)$ & $127.66(19)$ & $127.88(18)$ \\
\hline $\mathrm{Ln}-\mathrm{C} 1-\mathrm{C} 2-\mathrm{C} 3$, " & 176.79 & 176.72 & 177.10 & 177.38 & 177.49 \\
\hline Shortest Ln $\cdots \operatorname{Ln}, \AA$ & 6.305 & 6.324 & 6.290 & 6.284 & 6.287 \\
\hline
\end{tabular}


2.2. Single crystal structures of $2 \mathrm{Ho}-\mathrm{QN}, 3 \mathrm{Ho}-\mathrm{OPCy}$ and $4 \mathrm{Ho}-\mathrm{Lut}$
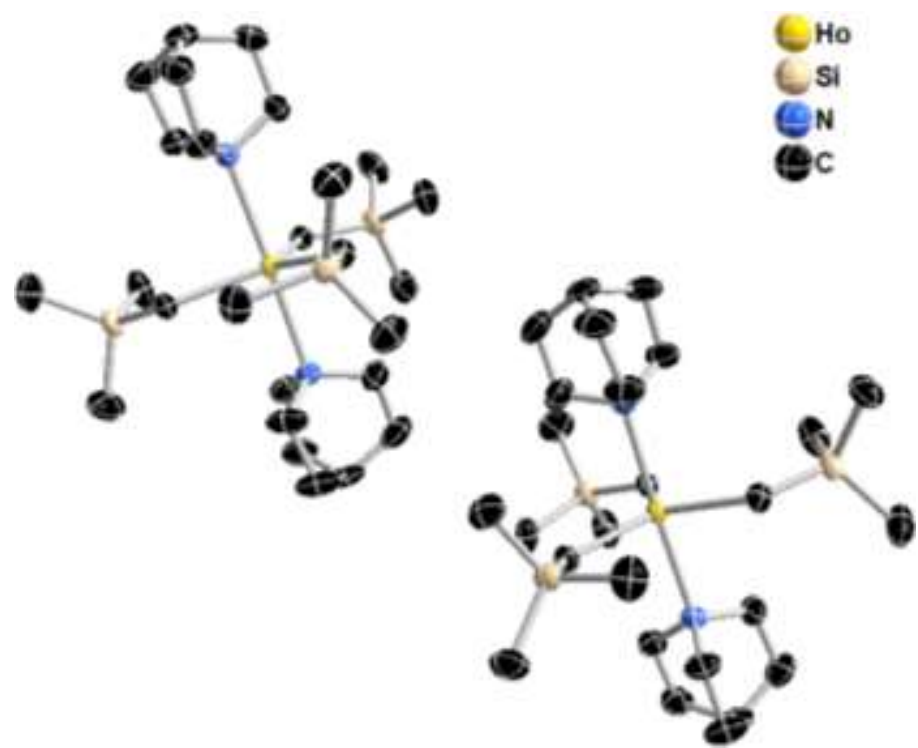

Figure S2. Molecular structures of $\mathbf{2 H o - Q N}$ in one asymmetry unit. Hydrogen atoms have been omitted for clarity.

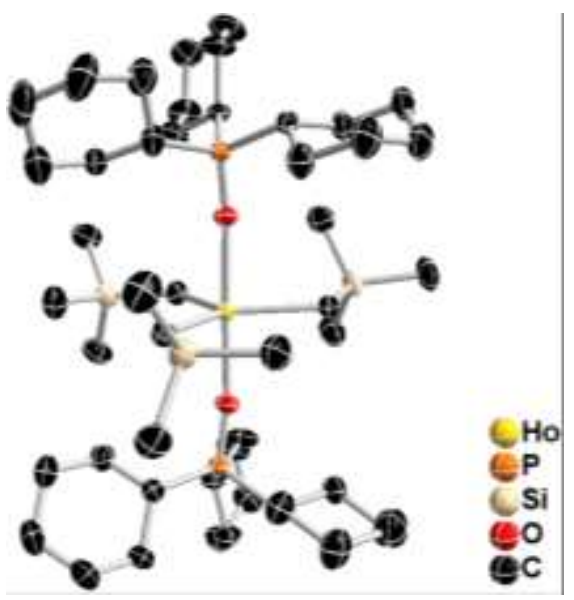

Figure S3. Molecular structures of $3 \mathrm{Ho}-\mathrm{OPC} \mathbf{y}_{3}$. Hydrogen atoms have been omitted for clarity.

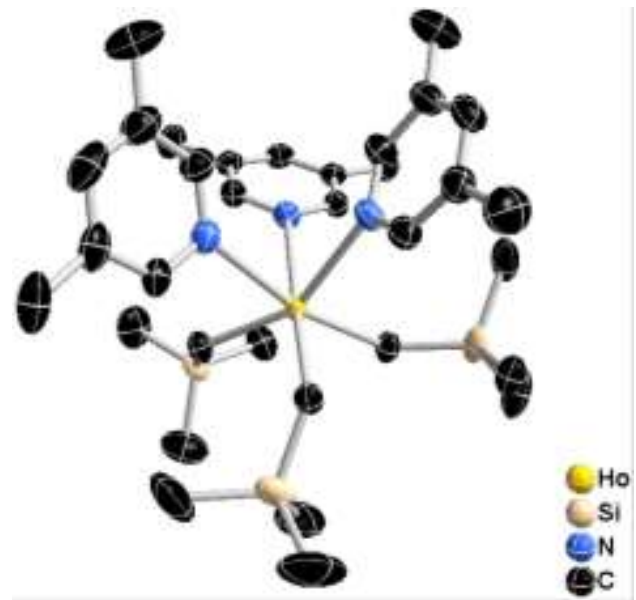

Figure S4. Molecular structures of $\mathbf{4 H o - L u t . ~ H y d r o g e n ~ a t o m s ~ h a v e ~ b e e n ~ o m i t t e d ~ f o r ~ c l a r i t y . ~}$ 
Table S3. Crystallographic data of 2Ho-QN, 3Ho-OPCy ${ }_{3}$ and $4 \mathrm{Ho}$-Lut

\begin{tabular}{|c|c|c|c|}
\hline Formula & $\begin{array}{c}\mathrm{C}_{26} \mathrm{H}_{59} \mathrm{~N}_{2} \mathrm{Si}_{3} \mathrm{Ho} \\
(2 \mathrm{Ho}-\mathrm{QN})\end{array}$ & $\begin{array}{c}\mathrm{C}_{48} \mathrm{H}_{99} \mathrm{O}_{2} \mathrm{Si}_{3} \mathrm{P}_{2} \mathrm{Ho} \\
\left.(3 \mathrm{Ho}-\mathrm{OPCy})_{3}\right)\end{array}$ & $\begin{array}{c}\mathrm{C}_{33} \mathrm{H}_{60} \mathrm{~N}_{3} \mathrm{Si}_{3} \mathrm{Ho} \\
\text { (4Ho-Lut) }\end{array}$ \\
\hline Formula Weight & 648.95 & 1019.41 & 748.04 \\
\hline$T, \mathrm{~K}$ & $180(2)$ & $180(2)$ & $180(2)$ \\
\hline$\lambda, \AA$ & 0.71073 & 0.71073 & 0.71073 \\
\hline Crystal System & Monoclinic & Orthorhombic & Monoclinic \\
\hline Space Group & $P_{2} / n$ & $P 2_{1} 2_{1} 2_{1}$ & $p_{2} / n$ \\
\hline$a, A$ & $10.5275(2)$ & $14.1728(2)$ & $13.5452(9)$ \\
\hline$b, A$ & $18.3330(4)$ & $18.2421(2)$ & $16.4751(4)$ \\
\hline$c, \bar{A}$ & $35.6271(7)$ & $21.6747(2)$ & $25.5876(16)$ \\
\hline $8 ;^{\circ}$ & $97.814(2)$ & 90 & $133.930(11)$ \\
\hline$z$ & 16 & 4 & 4 \\
\hline$v, A^{3}$ & $6812.2(2)$ & $5603.81(11)$ & $4112.3(7)$ \\
\hline$D_{v} \mathrm{~g} \mathrm{~cm}^{-3}$ & 1.265 & 1.208 & 1.208 \\
\hline$F(000)$ & 2704.0 & 2168.0 & 1552.0 \\
\hline$\mu, \mathrm{mm}^{-1}$ & 2.443 & 1.565 & 2.033 \\
\hline$R_{1}$ (reflections) & $0.0294(14225)$ & $0.0234(13086)$ & $0.0229(8769)$ \\
\hline$w R_{2}$ (reflections) & $0.0589(17274)$ & $0.0634(14133)$ & $0.0796(10377)$ \\
\hline$s$ & 1.035 & 1.101 & 1.136 \\
\hline
\end{tabular}

Table S4. Comparison of structural data for $1 \mathrm{Ho}-\mathrm{THF}, \mathbf{2 H o - Q N}$ and $3 \mathrm{Ho}-\mathrm{OPC} \mathbf{y}_{3}$

\begin{tabular}{|c|c|c|c|}
\hline & 1Ho-THF & $2 \mathrm{Ho}-\mathrm{QN}$ & $3 \mathrm{Ho}-\mathrm{OPC} \mathrm{y}_{3}$ \\
\hline \multirow{4}{*}{$\mathrm{Ho}-\mathrm{O} / \mathrm{N}(\bar{A})$} & $2.362(2)$ & $2.526(2) / 2.532(2)$ & $2.265(2)$ \\
\hline & & & \\
\hline & $2.352(2)$ & $2.542(2) / 2.549(2)$ & $2.259(2)$ \\
\hline & $2.389(3)$ & $2.405(2) / 2.401(2)$ & $2.417(3)$ \\
\hline \multirow[t]{2}{*}{$\mathrm{Ho}-\mathrm{C}(\AA)$} & $2.399(3)$ & $2.406(2) / 2.402(2)$ & $2.436(3)$ \\
\hline & $2.412(3)$ & $2.410(2) / 2.418(2)$ & $2.441(3)$ \\
\hline \multirow[t]{2}{*}{$\mathrm{O} / \mathrm{N}-\mathrm{Ho}-\mathrm{O} / \mathrm{N}\left({ }^{\circ}\right)$} & $177.79(7)$ & $177.73(7) / 178.53(7)$ & $176.86(8)$ \\
\hline & $109.99(10)$ & $119.62(8) / 118.16(9)$ & $115.81(13)$ \\
\hline \multirow[t]{2}{*}{$\mathrm{C}-\mathrm{Ho}-\mathrm{C}\left({ }^{\circ}\right)$} & $134.00(11)$ & $120.00(9) / 120.86(9)$ & $115.82(13)$ \\
\hline & $115.89(10)$ & $120.36(8) / 120.93(9)$ & $128.37(12)$ \\
\hline$d\left(\mathrm{Ho}_{\mathrm{o}}-P_{\mathrm{ccc}}\right)(\bar{A})$ & $0.0475(3)$ & $0.0218(5) / 0.0292(6)$ & $0.0123(4)$ \\
\hline Nearest Ho $\cdots$ Ho $(\AA)$ & $6.290(0)$ & $5.136(3)$ & $11.516(1)$ \\
\hline
\end{tabular}




\section{Magnetic Properties}

Samples were closely wrapped by two layers of para-films followed by being plugged into a capsule in glovebox, and then fixed in a straw. This straw was placed in a Schlenk tube before being transferred out of glovebox. Measurements of direct current (DC) susceptibilities under a static field of 1000 Oe and alteranative current (AC) susceptibilities with frequencies ranging from 1 to $1000 \mathrm{~Hz}$ were performed on a Quantum Design MPMS2 XL-5 SQUID magnetometer on polycrystalline samples. Measurements of AC susceptibilities with frequencies ranging from 100 to $10000 \mathrm{~Hz}$ was performed on a Quantum Design PPMS on polycrystalline samples. All DC magnetic susceptibilities were corrected for diamagnetic contribution from the sample holder, para-films, capsules and diamagnetic contributions from the molecule using Pascal's constants ${ }^{[4]}$.

\subsection{Magnetic dynamics of 1 Th-THF}
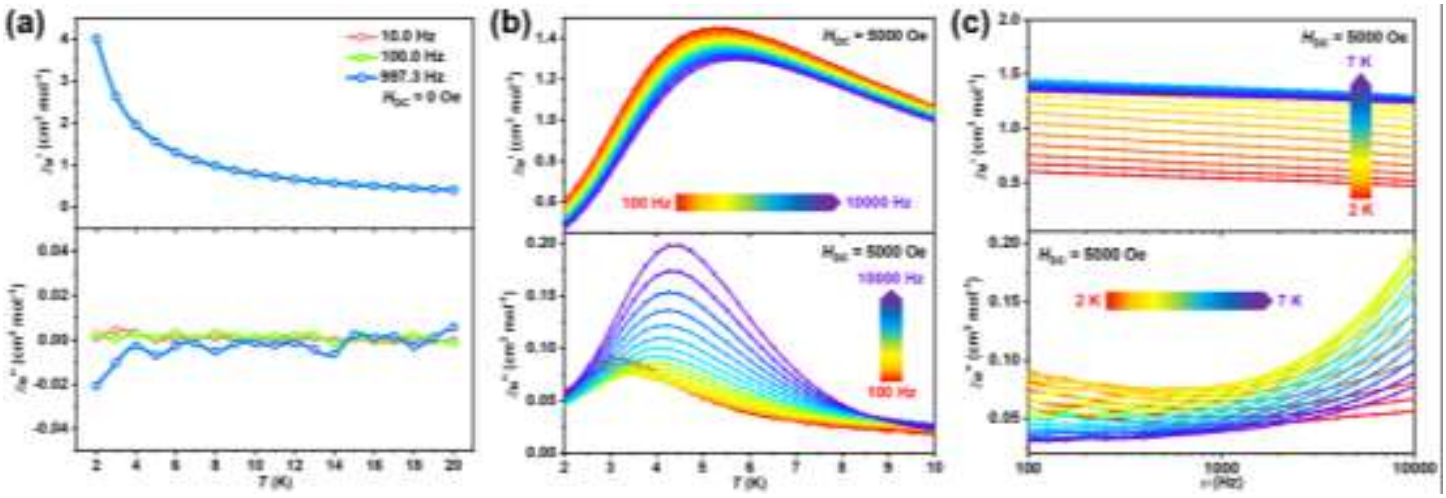

Figure S5. Temperature-dependence of molar AC susceptibilities (upper: $\chi_{\mathrm{M}}{ }^{\prime}$; lower: $\chi_{\mathrm{M}}{ }^{\prime \prime}$ ) for 1 Tb-THF under a zero applied DC field (a) and a 5000 Oe DC field (b, c).

\subsection{Magnetic dynamics of 1Dy-THF}
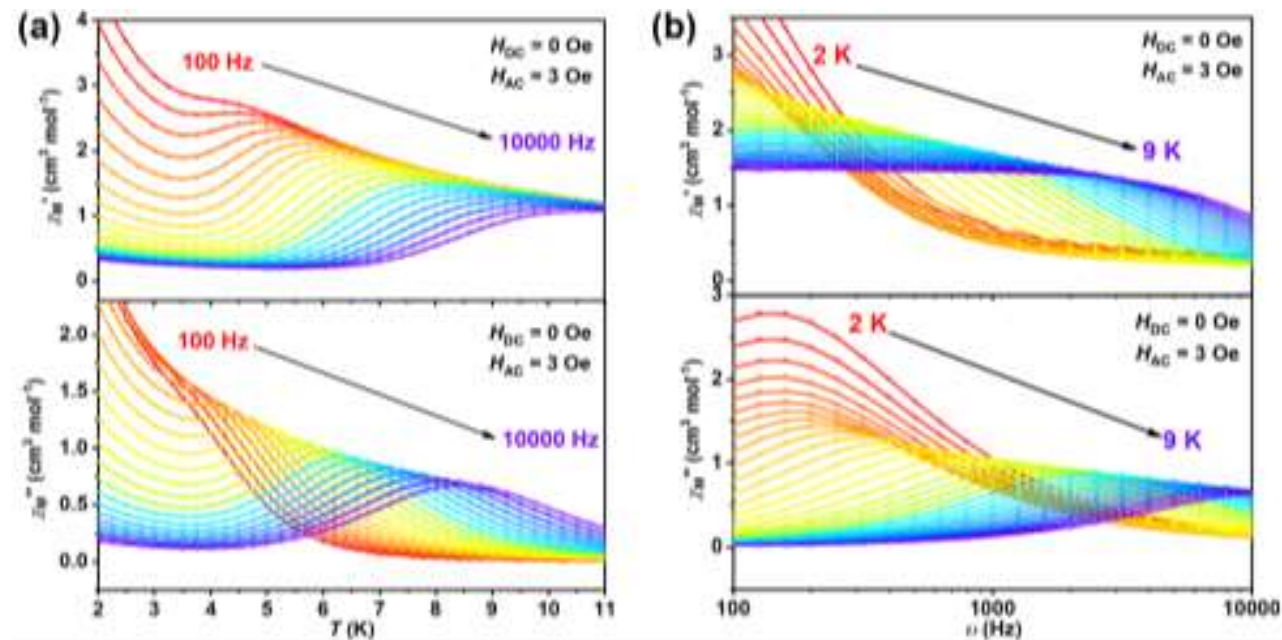

Figure S6. Temperature- (a) and frequency- (b) dependence of molar AC susceptibilities (upper: $\chi_{\mathrm{M}}{ }^{\prime}$; lower: $\chi_{\mathrm{M}}{ }^{\prime \prime}$ ) for 1Dy-THF under a zero applied DC field and certain AC frequencies from 100 to $10000 \mathrm{~Hz}$. 

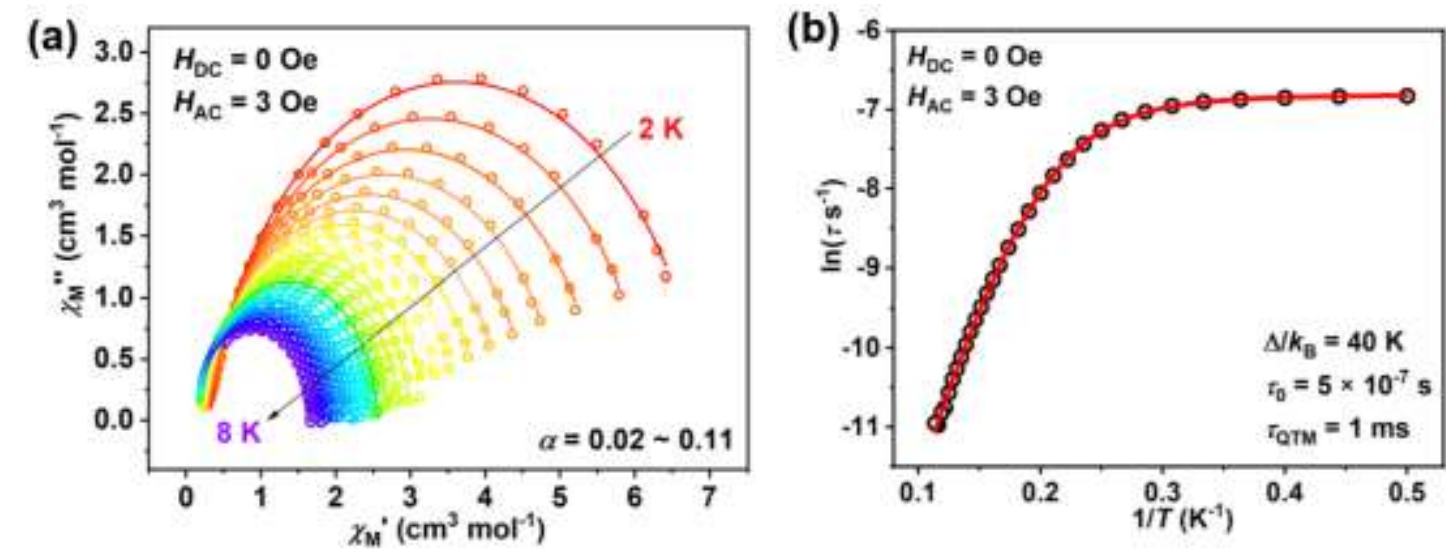

Figure S7. (a) Argand plots of $\chi_{M}{ }^{\prime \prime} v s \chi_{M}$ for 1Dy-THF under a zero applied DC magnetic field. (b) Plot of natural log of relaxation time vs $1 / T$. The red solid line represents the best fitting using a combination of Orbach, Raman and QTM process $\left(\tau^{-1}=\tau_{0}^{-1} \exp \left(-\Delta / k_{\mathrm{B}} T\right)+C T^{n}+\tau_{\mathrm{QTM}}{ }^{-1}\right)$. The fitting gives relaxation barrier $\Delta / k_{\mathrm{B}}=40 \mathrm{~K}$ and $\tau_{0}=5 \times 10^{-7} \mathrm{~s}$.

Table S5. Fitting parameters for the plot of $\ln \tau^{\sim 1 / T}$ using $\tau^{-1}=\tau_{0}^{-1} \exp \left(-\Delta / k_{\mathrm{B}} T\right)+C T^{n}+\tau_{\mathrm{QTM}}{ }^{-1}$ for 1Dy-THF.

\begin{tabular}{cccc}
\hline & Value & Standard Error & Dependency \\
\hline$\tau_{0}$ & $5.41111 \mathrm{E}-7$ & $4.55808 \mathrm{E}-7$ & 0.99935 \\
$\Delta / \boldsymbol{k}_{\mathrm{B}}$ & 40.30343 & 4.7218 & 0.99904 \\
$\boldsymbol{C}$ & 0.14458 & 0.07633 & 0.99966 \\
$n$ & 5.79899 & 0.30402 & 0.99973 \\
$\boldsymbol{\tau}_{\text {Qтм }}$ & 0.0011 & $1.95935 \mathrm{E}-5$ & 0.68445 \\
\hline
\end{tabular}

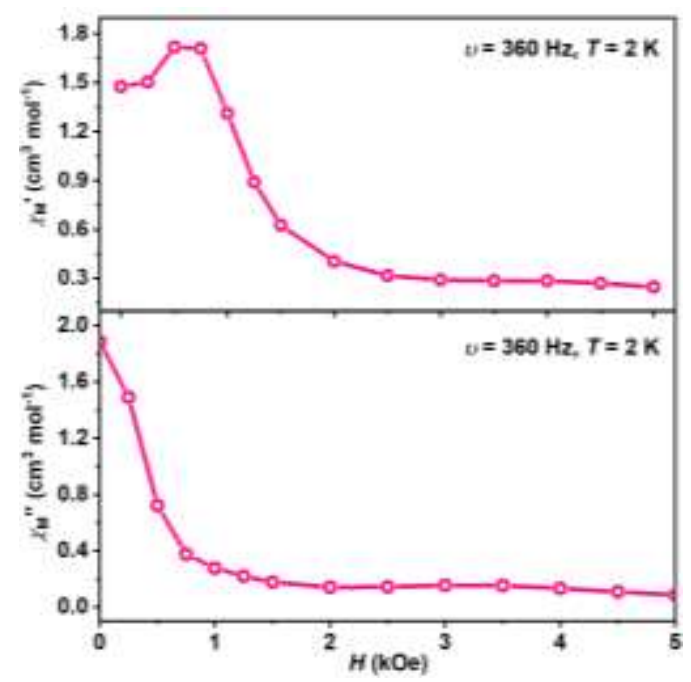

Figure S8. Plot of in-phase $\left(\chi_{\mathrm{M}^{\prime}}\right.$, upper) and out-of-phase $\left(\chi_{\mathrm{M}}{ }^{\prime \prime}\right.$, lower) molar AC susceptibility for 1Dy-THF vs applied DC fields under $A C$ magnetic field with oscillation frequency of $360 \mathrm{~Hz}$ at $2 \mathrm{~K}$. 

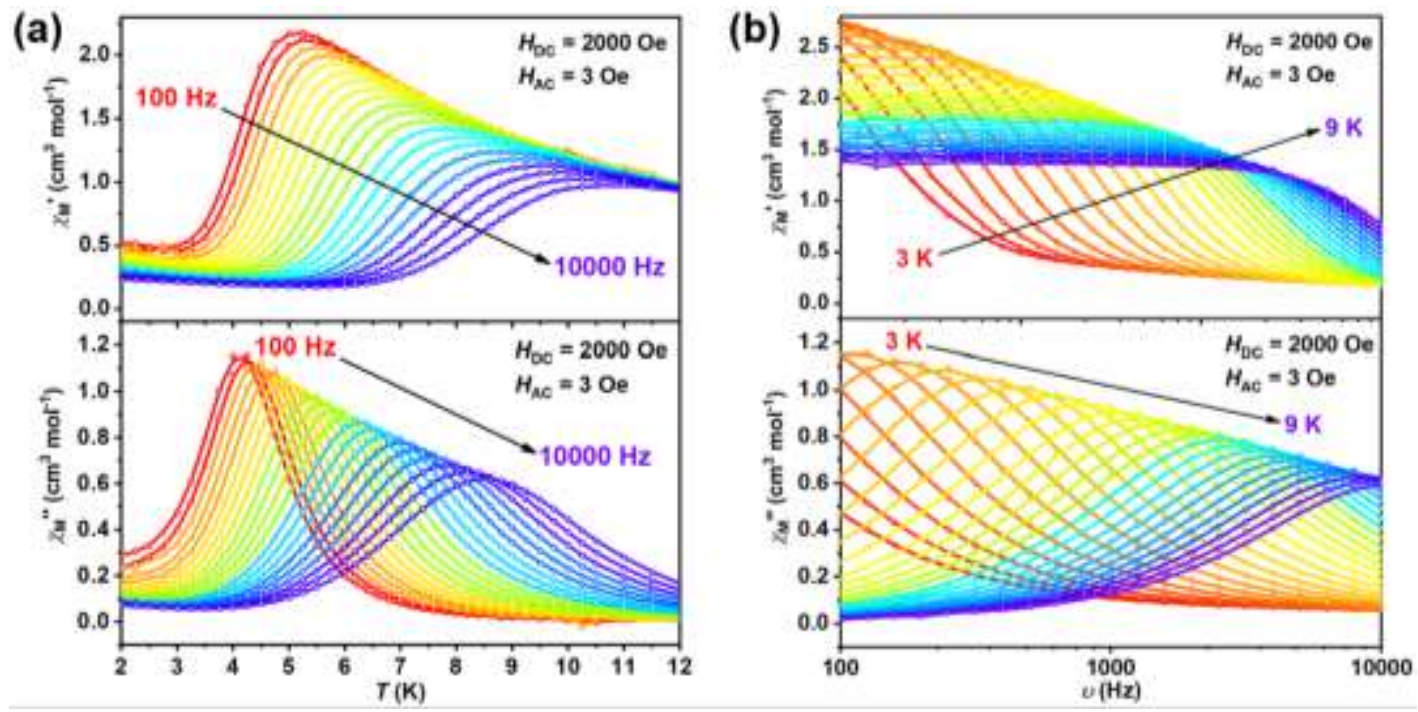

Figure S9. Temperature- (a) and frequency- (b) dependence of molar AC susceptibilities (upper: $\chi_{\mathrm{M}}{ }^{\prime}$; lower: $\chi_{\mathrm{M}}{ }^{\prime \prime}$ ) for 1Dy-THF under a 2000 Oe applied DC field and certain AC frequencies from 100 to $10000 \mathrm{~Hz}$.
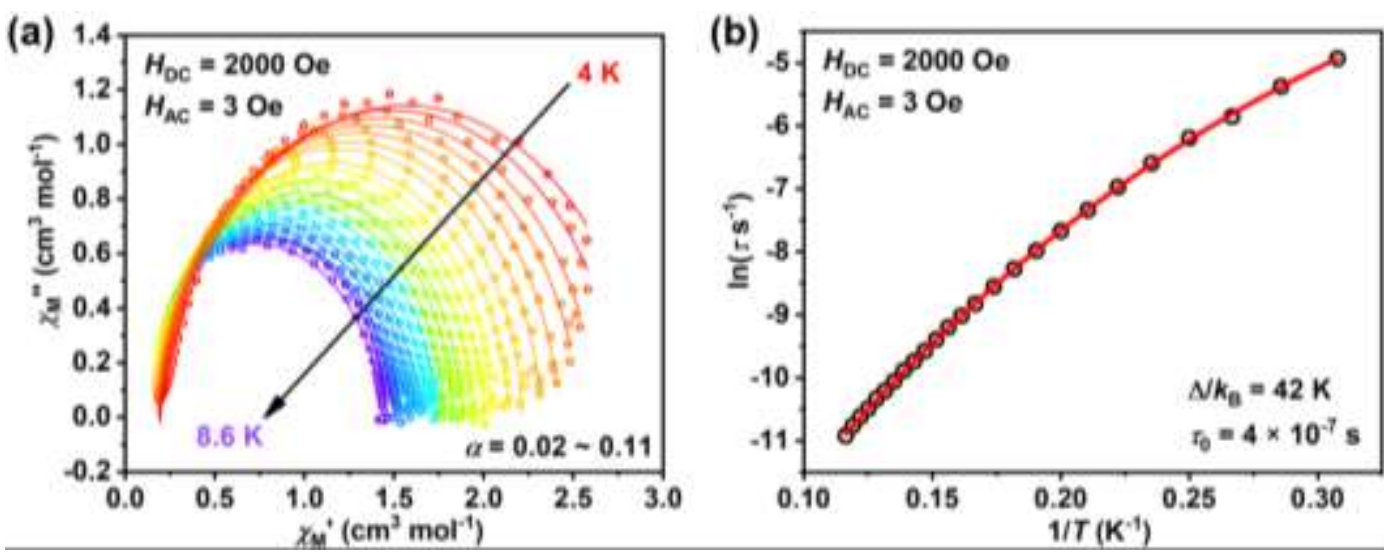

Figure S10. (a) Argand plots of $\chi_{\mathrm{M}}{ }^{\prime \prime}$ vs $\chi_{\mathrm{M}}{ }^{\prime}$ for 1Dy-THF under a 2000 Oe applied DC magnetic field. (b) Plot of natural $\log$ of relaxation time $v s 1 / T$. The red solid line represents the best fitting using a combination of Orbach and Raman $\left(\tau^{-1}=\tau_{0}^{-1} \exp \left(-\Delta / k_{\mathrm{B}} T\right)+C T^{n}\right)$. The fitting gives relaxation barrier $\Delta / k_{\mathrm{B}}=42 \mathrm{~K}$ and $\tau_{0}=4 \times 10^{-7} \mathrm{~s}$.

Table S6. Fitting parameters for the plot of $\ln \tau^{\sim} 1 / T$ using $\tau^{-1}=\tau_{0}^{-1} \exp \left(-\Delta / k_{B} T\right)+C T^{n}$ under a 2000 Oe of DC field for 1Dy-THF.

\begin{tabular}{cccc}
\hline & Value & Standard Error & Dependency \\
\hline$\tau_{0}$ & $3.99766 \mathrm{E}-7$ & $8.14216 \mathrm{E}-8$ & 0.9977 \\
$\Delta / \boldsymbol{k}_{\mathrm{B}}$ & 41.87343 & 0.75542 & 0.99296 \\
$\boldsymbol{C}$ & 0.15618 & 0.01716 & 0.9985 \\
$\boldsymbol{n}$ & 5.71775 & 0.08668 & 0.99919 \\
\hline
\end{tabular}




\subsection{Magnetic dynamics of $1 \mathrm{Ho}-\mathrm{THF}$}
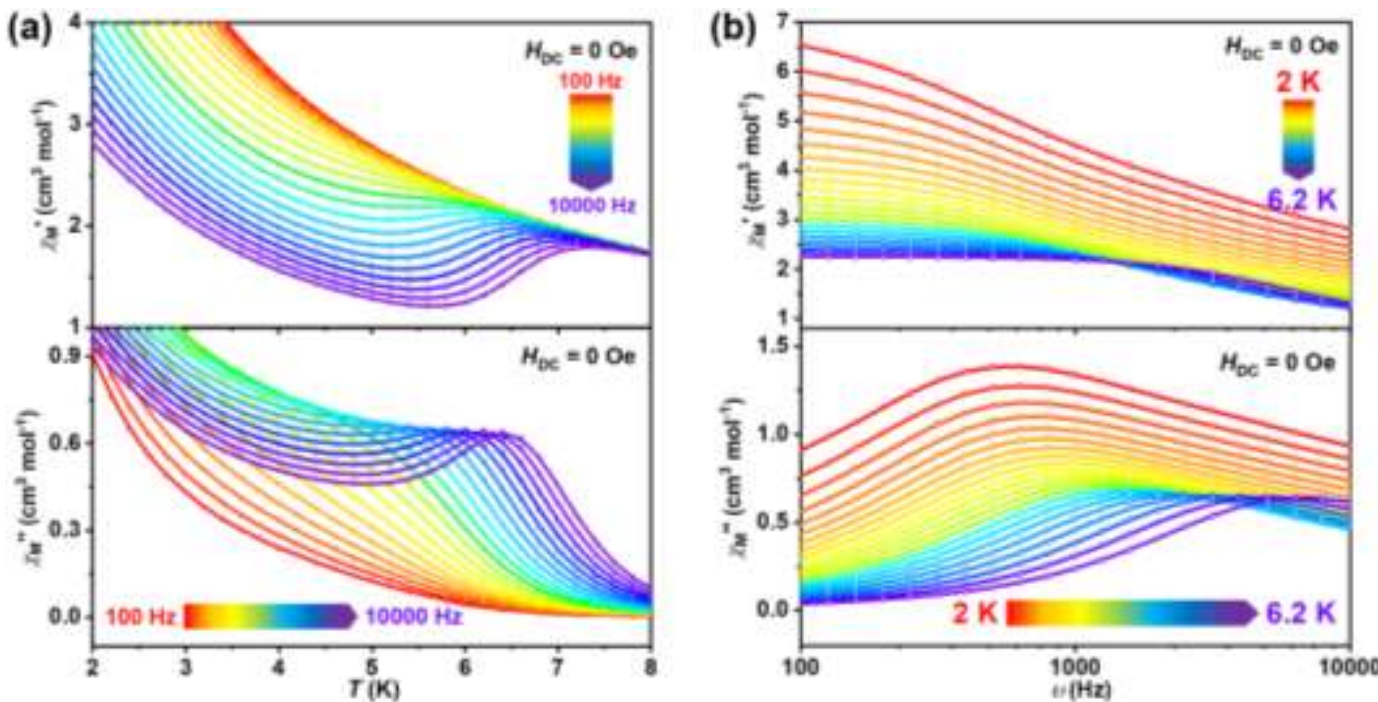

Figure S11. Temperature- (a) and frequency- (b) dependence of molar AC susceptibilities (upper: $\chi_{\mathrm{M}}{ }^{\prime}$; lower: $\chi_{\mathrm{M}}{ }^{\prime \prime}$ ) for $\mathbf{1 H o - T H F}$ under a zero applied DC field and certain AC frequencies from 100 to $10000 \mathrm{~Hz}$.

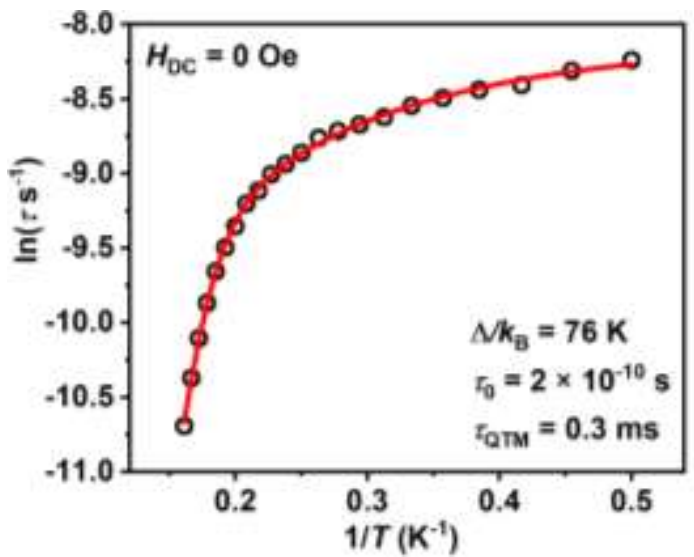

Figure S12. Plot of natural log of relaxation time vs $1 / T$ at zero field for $\mathbf{1 H o - T H F}$. The red solid line represents the best fitting using a combination of Orbach, Raman and QTM process $\left(\tau^{-1}=\tau_{0}^{-1} \exp \left(-\Delta / k_{\mathrm{B}} T\right)+C T^{n}+\tau_{\mathrm{QTM}}{ }^{-1}\right)$. The fitting gives relaxation barrier $\Delta / k_{\mathrm{B}}=76 \mathrm{~K}$ and $\tau_{0}=2 \times 10^{-10} \mathrm{~s}$.

Table S7. Fitting parameters for the plot of $\ln \tau^{\sim} 1 / T$ using $\tau^{-1}=\tau_{0}^{-1} \exp \left(-\Delta / k_{\mathrm{B}} T\right)+C T^{n}+\tau_{\mathrm{QTM}}{ }^{-1}$ at zero field for 1 HoTHF.

\begin{tabular}{cccc}
\hline & Value & Standard Error & Dependency \\
\hline$\tau_{0}$ & $1.69494 \mathrm{E}-10$ & $1.0928 \mathrm{E}-10$ & 0.9993 \\
$\Delta / \boldsymbol{k}_{\mathrm{B}}$ & 75.76586 & 4.078 & 0.99938 \\
$\boldsymbol{C}$ & 170.86432 & 65.11491 & 0.99941 \\
$\boldsymbol{n}$ & 2.27158 & 0.23635 & 0.99928 \\
$\boldsymbol{\tau}_{\mathrm{QTM}}$ & $3.25684 \mathrm{E}-4$ & $2.38721 \mathrm{E}-5$ & 0.9845 \\
\hline
\end{tabular}



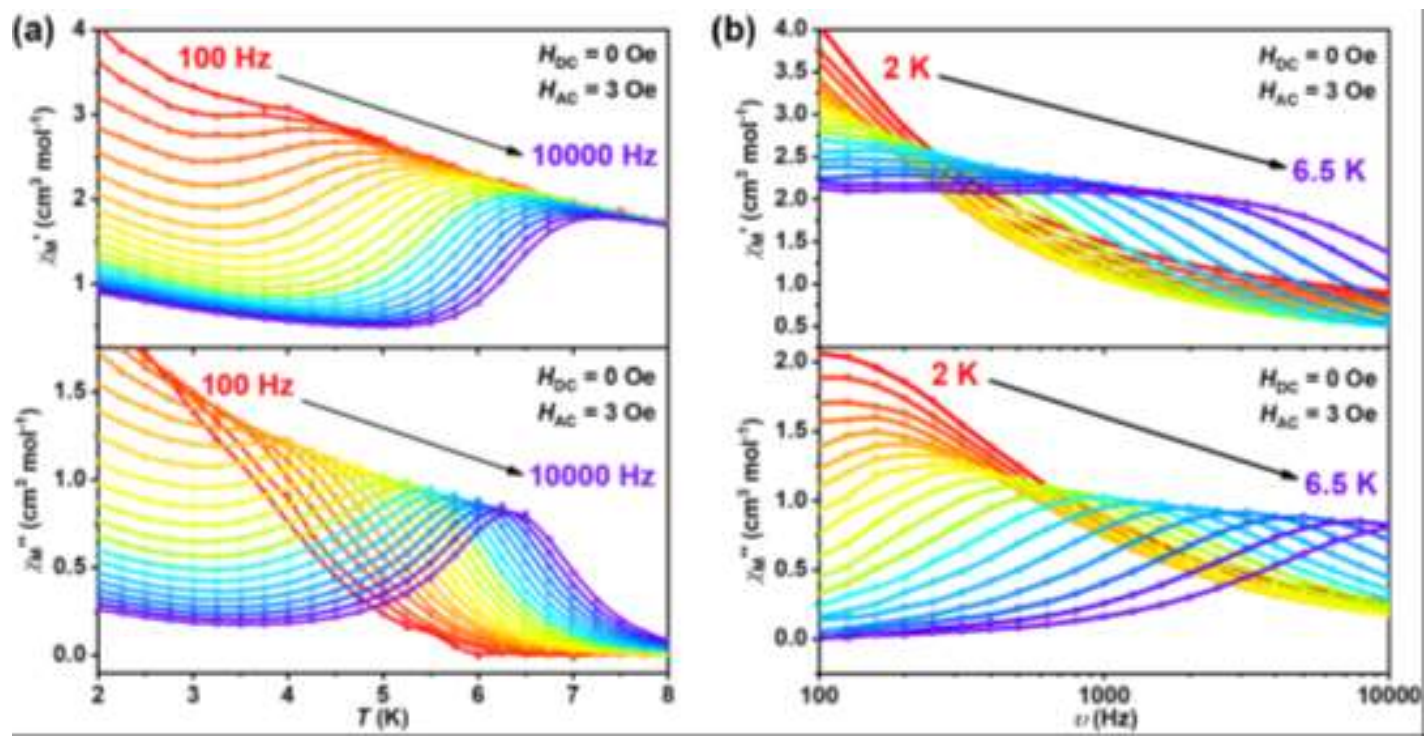

Figure S13. Temperature- (a) and frequency- (b) dependence of molar AC susceptibilities (upper: $\chi_{\mathrm{M}}{ }^{\prime}$; lower: $\chi_{\mathrm{M}}{ }^{\prime \prime}$ ) for 1Ho-THF@1Y-THF (1:20 dilution) under a zero applied DC field and certain AC frequencies from 100 to 10000 $\mathrm{Hz}$.

Table S8. Fitting parameters for the plot of $\ln \tau^{\sim} 1 / T$ using $\tau^{-1}=\tau_{0}^{-1} \exp \left(-\Delta / k_{B} T\right)+C T^{n}$ at zero field for 1Ho-THF@1YTHF.

\begin{tabular}{cccc}
\hline & Value & Standard Error & Dependency \\
\hline $\boldsymbol{\tau}_{\mathbf{0}}$ & $2.78897 \mathrm{E}-10$ & $2.9536 \mathrm{E}-10$ & 0.9976 \\
$\Delta / \boldsymbol{k}_{\mathrm{B}}$ & 72.06099 & 6.40325 & 0.99779 \\
$\boldsymbol{C}$ & 53.45593 & 12.80222 & 0.98709 \\
$\boldsymbol{n}$ & 2.65671 & 0.19512 & 0.9892 \\
\hline
\end{tabular}

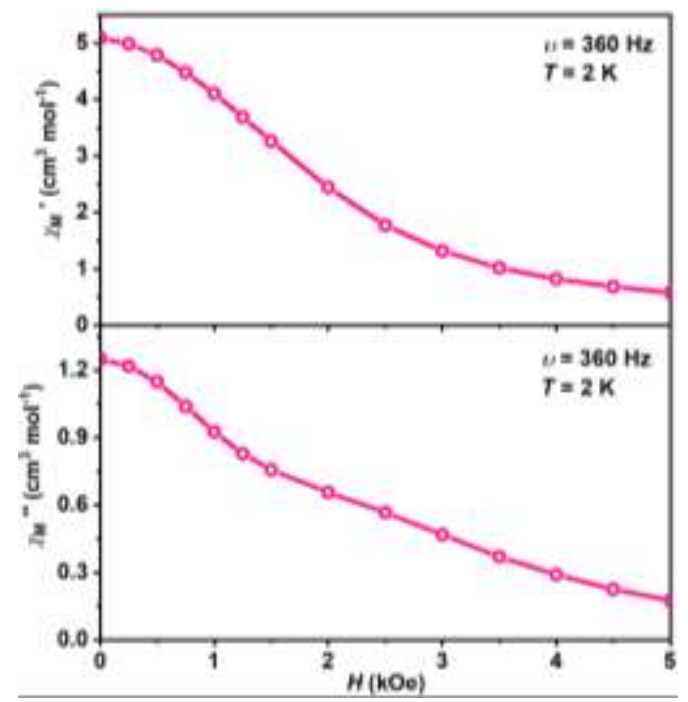

Figure S14. Plot of in-phase $\left(\chi_{\mathrm{M}^{\prime}}\right.$, upper) and out-of-phase ( $\chi_{\mathrm{M}}{ }^{\prime \prime}$, lower) molar AC susceptibilities for 1Ho-THF vs applied DC fields under $A C$ magnetic field with oscillation frequency of $360 \mathrm{~Hz}$ at $2 \mathrm{~K}$. 

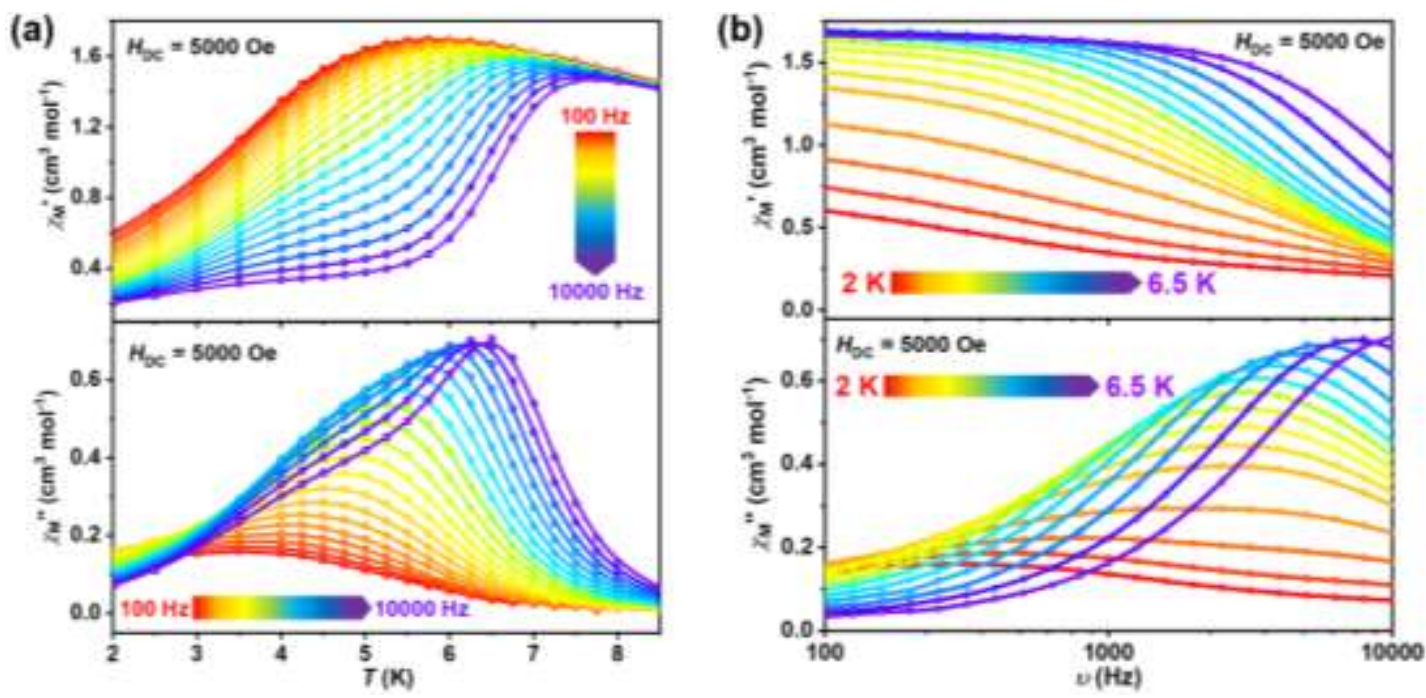

Figure S15. Temperature- (a) and frequency- (b) dependence of molar AC susceptibilities (upper: $\chi_{\mathrm{M}}{ }^{\prime}$; lower: $\chi_{\mathrm{M}}{ }^{\prime \prime}$ ) for $\mathbf{1 H o - T H F ~ u n d e r ~ a ~} 5000$ Oe applied DC field and certain AC frequencies from 100 to $10000 \mathrm{~Hz}$.

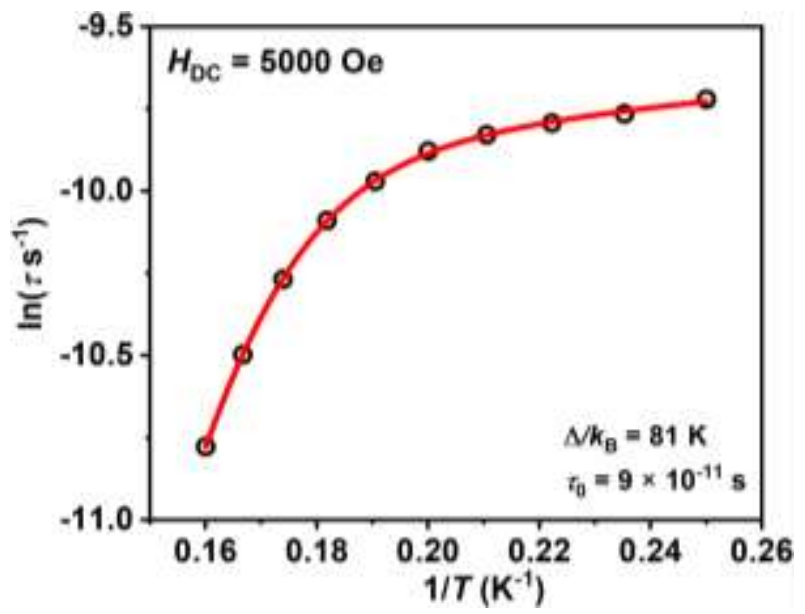

Figure S16. Plot of natural log of relaxation time vs $1 / T$ for $\mathbf{1 H o - T H F}$ under a 5000 Oe DC field. The red solid line represents the best fitting using a combination of Orbach and Raman process $\left(\tau^{-1}=\tau_{0}^{-1} \exp \left(-\Delta / k_{\mathrm{B}} T\right)+C T^{n}\right)$. The fitting gives relaxation barrier $\Delta / k_{\mathrm{B}}=81 \mathrm{~K}$ and $\tau_{0}=9 \times 10^{-11} \mathrm{~s}$. 

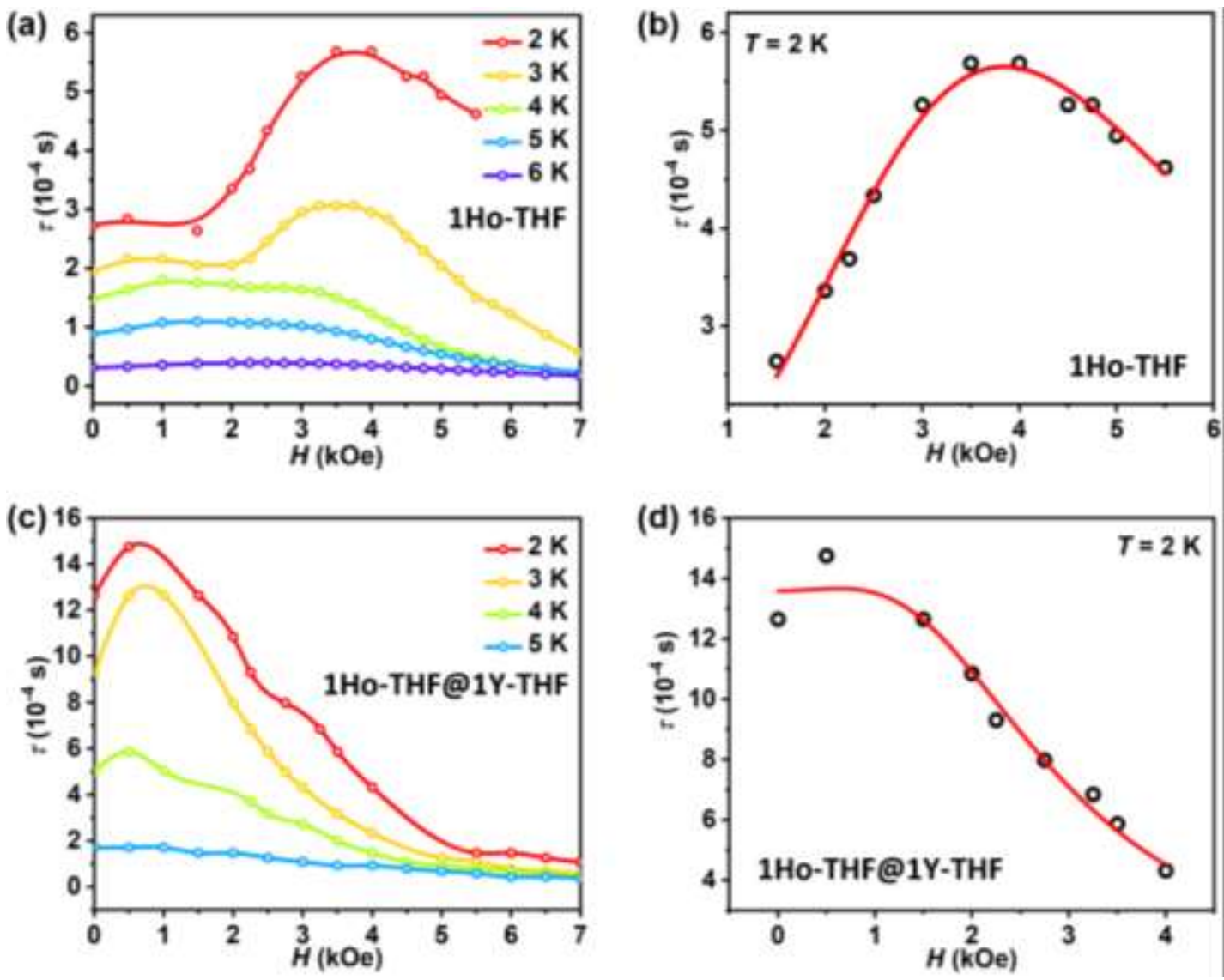

Figure S17. DC field-dependence of relaxation time $\tau$ at different temperatures for 1Ho-THF (a) and 1Ho-THF@1YTHF (c), respectively. At $T=2 \mathrm{~K}$, the data of $\tau$ versus $H$ for 1Ho-THF (b) and 1Ho-THF@1Y-THF (d) was fitted by $\tau^{-1}=$ $A H^{2}+B /\left(1+C H^{2}\right)$. Black circles are data extracted from the measurements, and red solid line represents the best fitting.

Table S9. Fitting parameters for the plot of $\tau^{\sim} H$ at $2 \mathrm{~K}$ using $\tau^{-1}=A H^{2}+B /\left(1+C H^{2}\right)$ for 1 Ho-THF and 1Ho-THF@1YTHF, respectively.

\begin{tabular}{cccc|ccc}
\hline \multicolumn{3}{c|}{ 1Ho-THF } & \multicolumn{3}{c}{ 1Ho-THF@1Y-THF } \\
\hline & Value & Standard Error & Dependency & Value & Standard Error & Dependency \\
\hline A & 0.00567 & $1.74071 \mathrm{E}-4$ & 0.70662 & 0.01275 & 0.00121 & 0.74677 \\
B & 0.91028 & 0.15078 & 0.99237 & 0.07356 & 0.0029 & 0.3396 \\
C & 0.5936 & 0.12959 & 0.99339 & 0.20197 & 0.075 & 0.79454 \\
\hline
\end{tabular}


3.4. Magnetic dynamics of $2 \mathrm{Ho}-\mathrm{QN}$
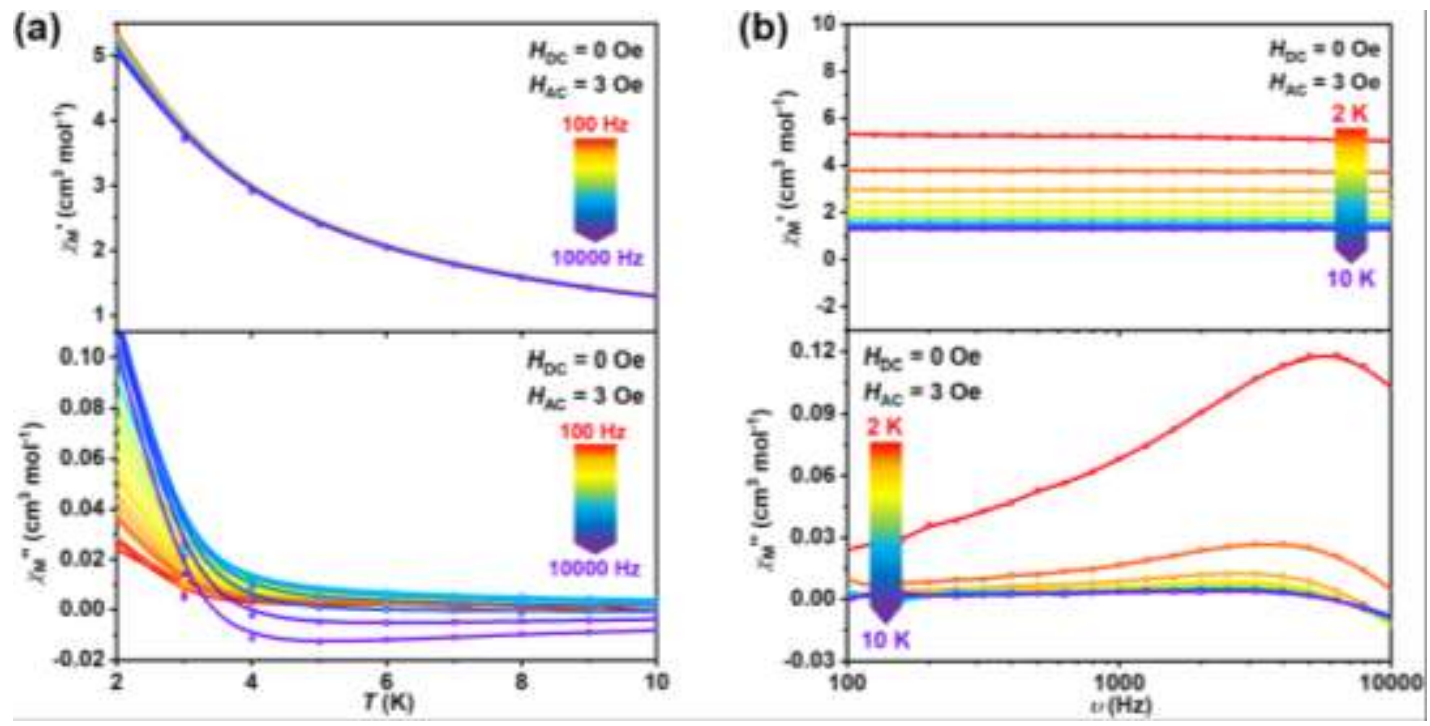

Figure S18. Temperature- (a) and frequency- (b) dependence of molar AC susceptibilities (upper: $\chi_{\mathrm{M}^{\prime}}$; lower: $\chi_{\mathrm{M}}{ }^{\prime \prime}$ ) for $\mathbf{2 H o - Q N}$ under a zero applied DC field and certain AC frequencies from 100 to $10000 \mathrm{~Hz}$.

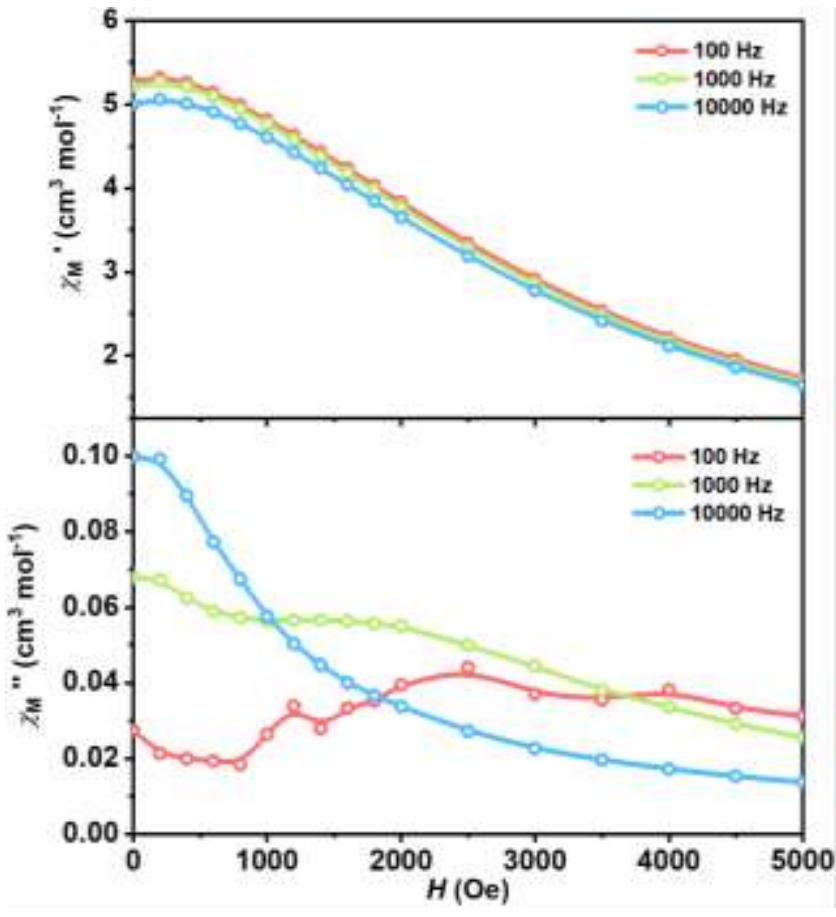

Figure S19. Plot of in-phase $\left(\chi_{\mathrm{M}}{ }^{\prime}\right.$, upper) and out-of-phase ( $\chi_{\mathrm{M}}{ }^{\prime \prime}$, lower) molar AC susceptibilities for 2Ho-QN vs applied DC fields under AC magnetic field with oscillation frequency of $100,1000,10000 \mathrm{~Hz}$ at $2 \mathrm{~K}$. 

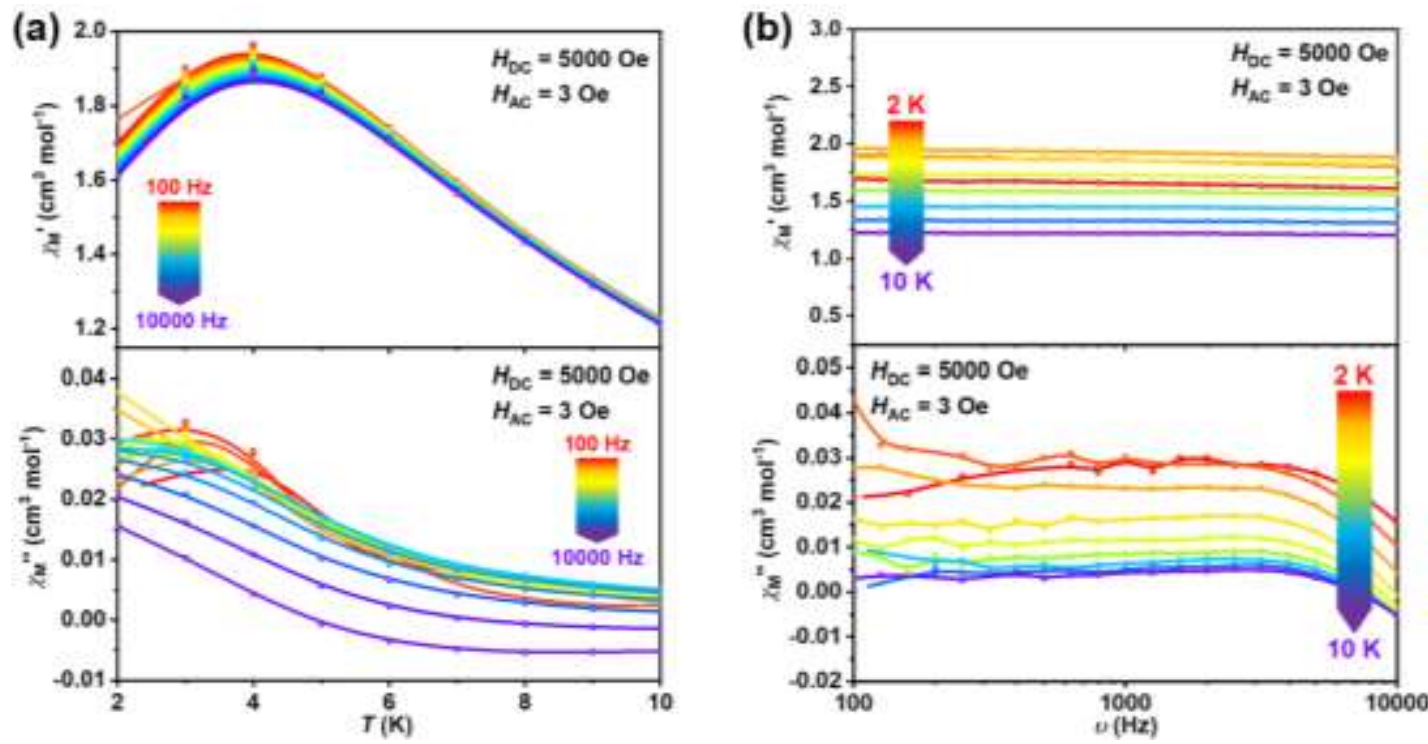

Figure S20. Temperature- (a) and frequency- (b) dependence of molar AC susceptibilities (upper: $\chi_{\mathrm{M}}{ }^{\prime}$; lower: $\chi_{\mathrm{M}}{ }^{\prime \prime}$ ) for $\mathbf{2 H o - Q N}$ under a 5000 Oe of DC field and certain AC frequencies from 100 to $10000 \mathrm{~Hz}$.

\subsection{Magnetic dynamics of $3 \mathrm{Ho}-\mathrm{OPC} y_{3}$}
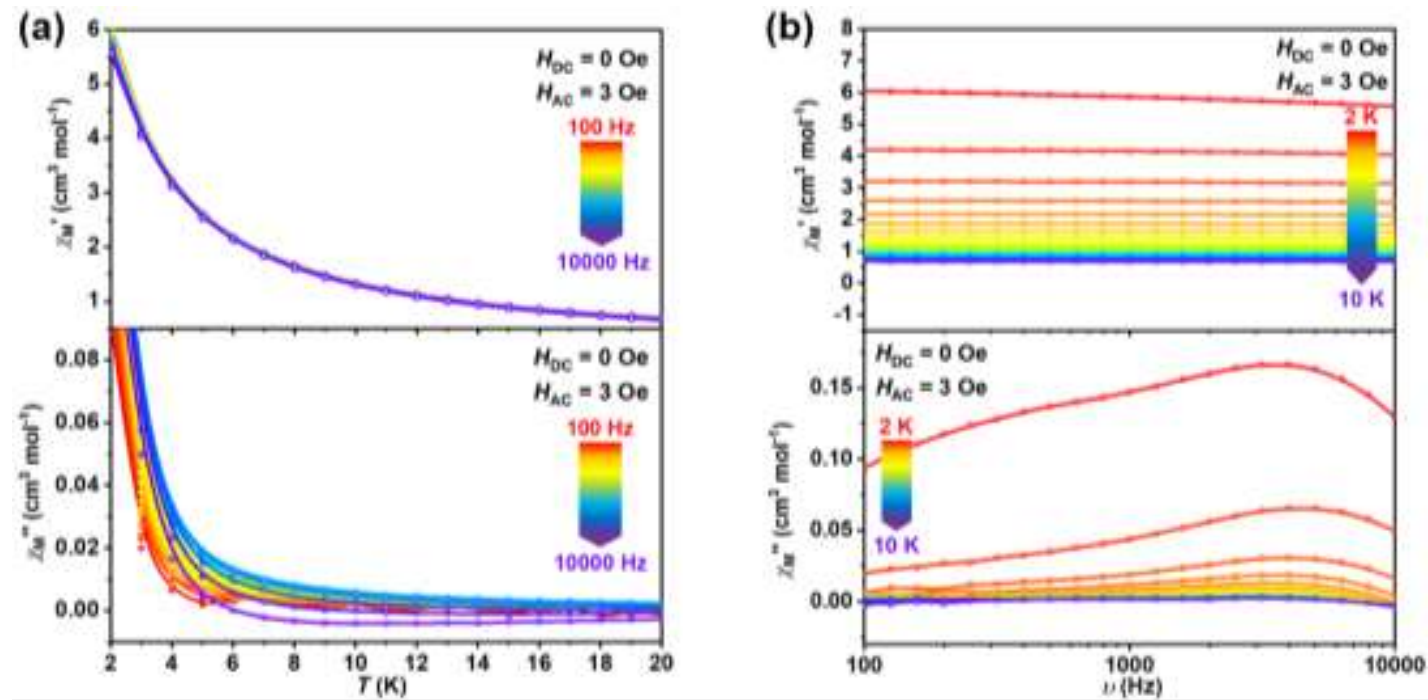

Figure S21. Temperature- (a) and frequency- (b) dependence of molar AC susceptibilities (upper: $\chi_{\mathrm{M}}{ }^{\prime}$; lower: $\chi_{\mathrm{M}}{ }^{\prime \prime}$ ) for $3 \mathrm{Ho}-\mathrm{OPC} \mathbf{y}_{3}$ under a zero applied DC field and certain AC frequencies from 100 to $10000 \mathrm{~Hz}$. 


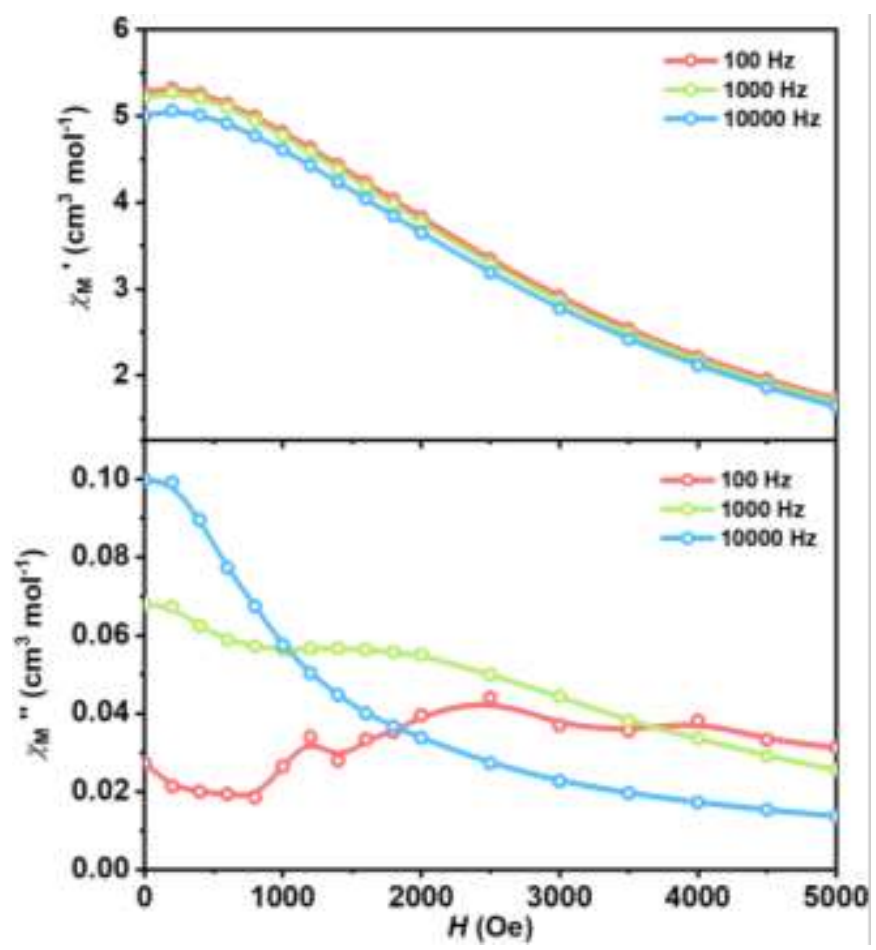

Figure S22. Plot of in-phase $\left(\chi_{\mathrm{M}}{ }^{\prime}\right.$, upper) and out-of-phase $\left(\chi_{\mathrm{M}}{ }^{\prime \prime}\right.$, lower) molar AC susceptibilities for $\mathbf{3 H o - O P C y _ { 3 }}$ vs applied DC fields under AC magnetic field with oscillation frequency of 100,1000, $10000 \mathrm{~Hz}$ at $2 \mathrm{~K}$.
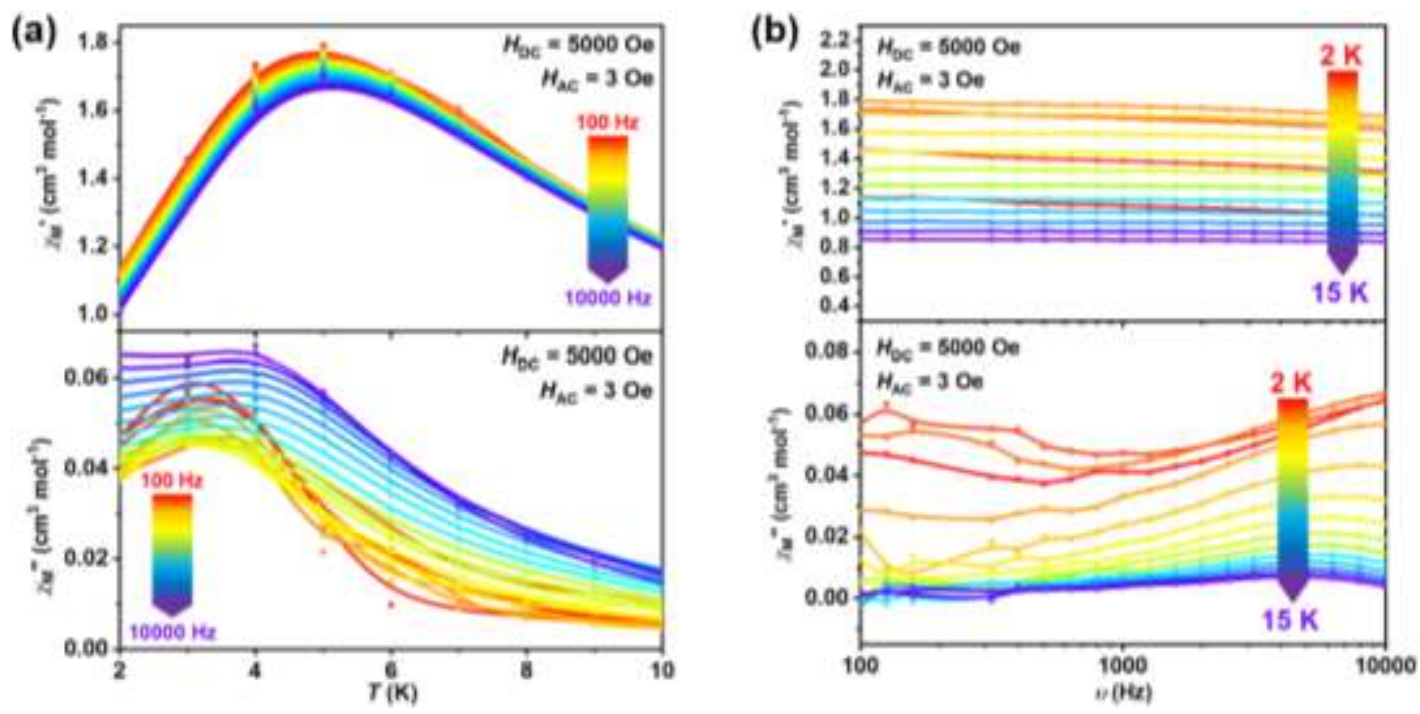

Figure S23. Temperature- (a) and frequency- (b) dependence of molar AC susceptibilities (upper: $\chi_{\mathrm{M}}{ }^{\prime}$; lower: $\chi_{\mathrm{M}}{ }^{\prime \prime}$ ) for $3 \mathrm{Ho}-\mathrm{OPC} \mathbf{y}_{3}$ under a $5000 \mathrm{Oe}$ of DC field and certain AC frequencies from 100 to $10000 \mathrm{~Hz}$. 
3.6. Magnetic dynamics of $4 \mathrm{Ho}$-Lut
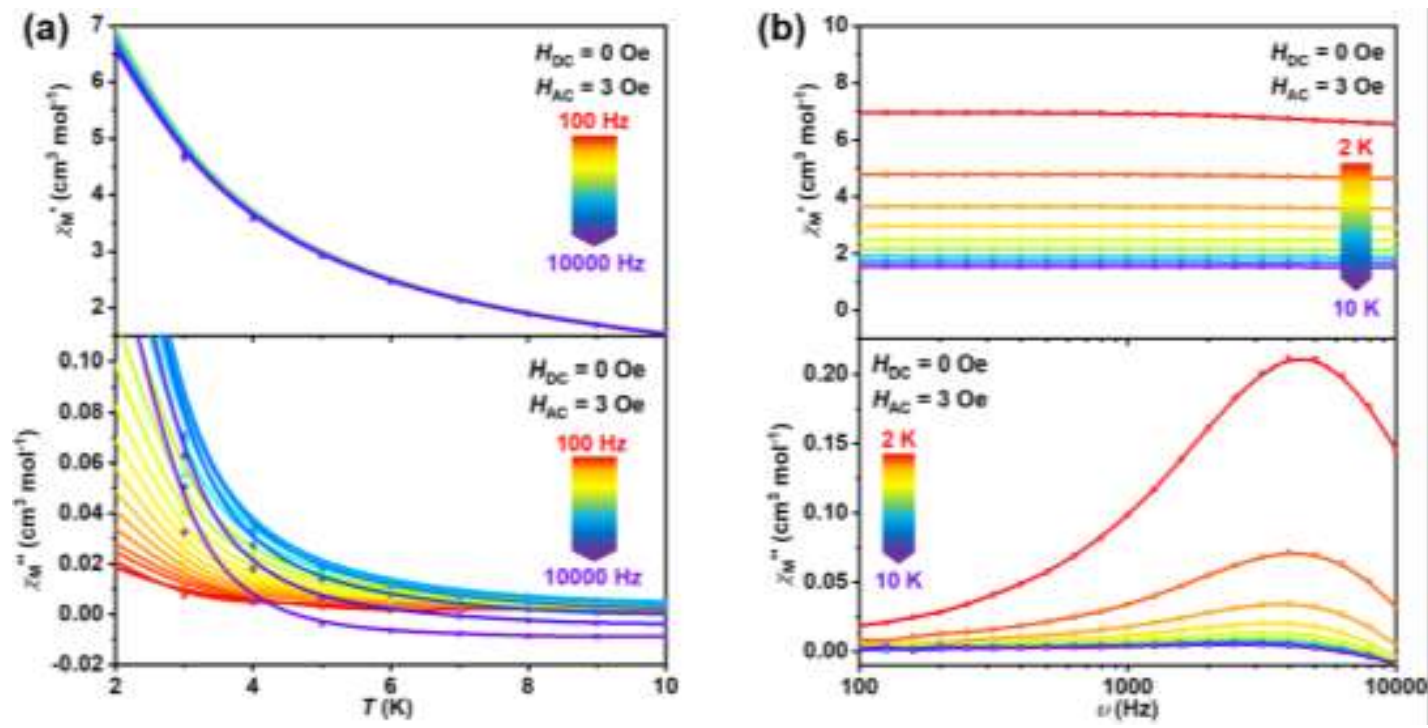

Figure S24. Temperature- (a) and frequency- (b) dependence of molar AC susceptibilities (upper: $\chi_{\mathrm{M}^{\prime}}$; lower: $\chi_{\mathrm{M}}{ }^{\prime \prime}$ ) for $\mathbf{4 H o - L u t ~ u n d e r ~ a ~ z e r o ~ a p p l i e d ~ D C ~ f i e l d ~ a n d ~ c e r t a i n ~ A C ~ f r e q u e n c i e s ~ f r o m ~} 100$ to $10000 \mathrm{~Hz}$.

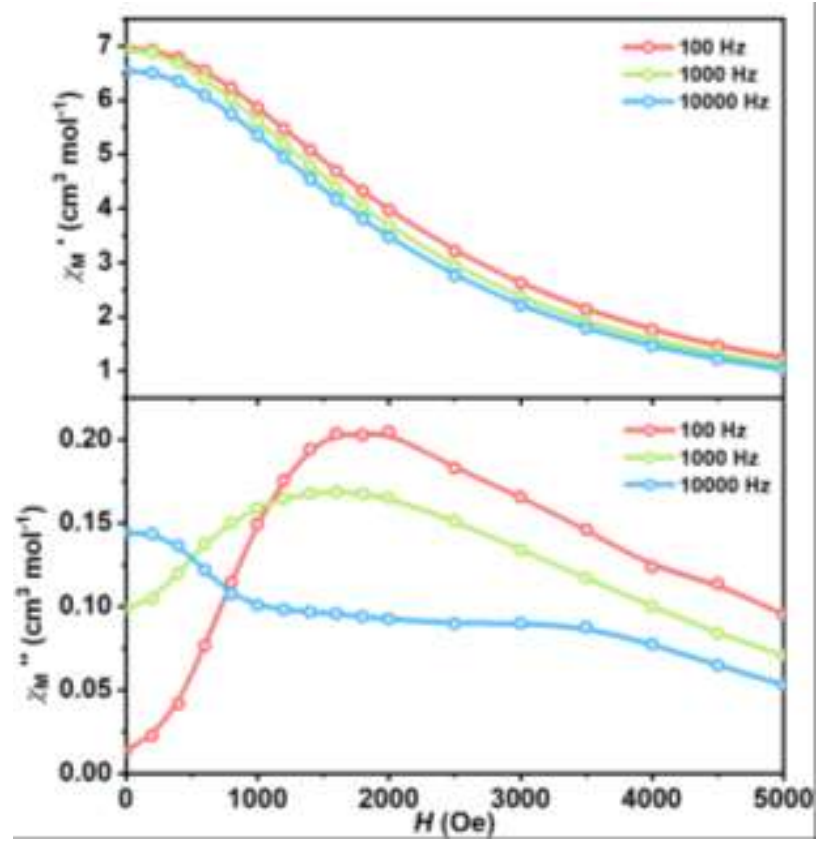

Figure S25. Plot of in-phase $\left(\chi_{\mathrm{M}}^{\prime}\right.$, upper) and out-of-phase $\left(\chi_{\mathrm{M}}{ }^{\prime \prime}\right.$, lower) molar AC susceptibilities for 4Ho-Lut vs applied DC fields under AC magnetic field with oscillation frequency of 100,1000, $10000 \mathrm{~Hz}$ at $2 \mathrm{~K}$. 
(a)

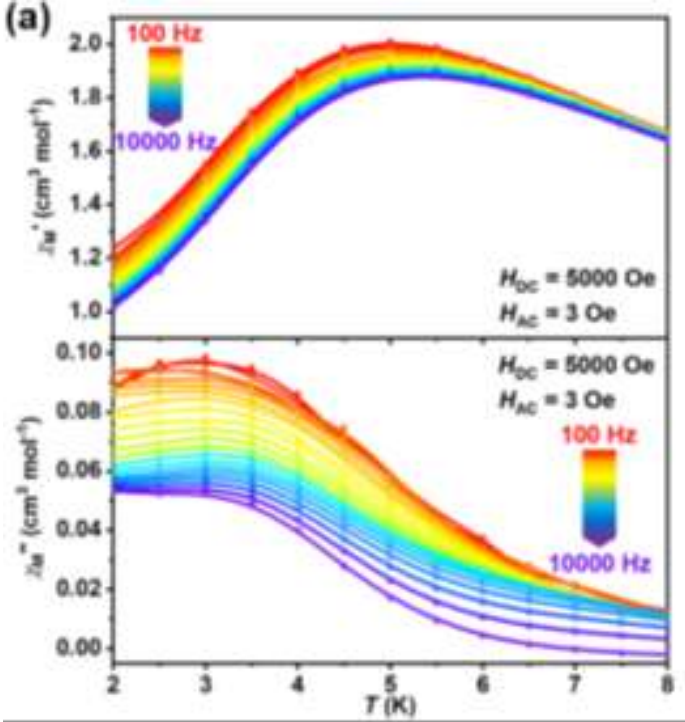

(b)

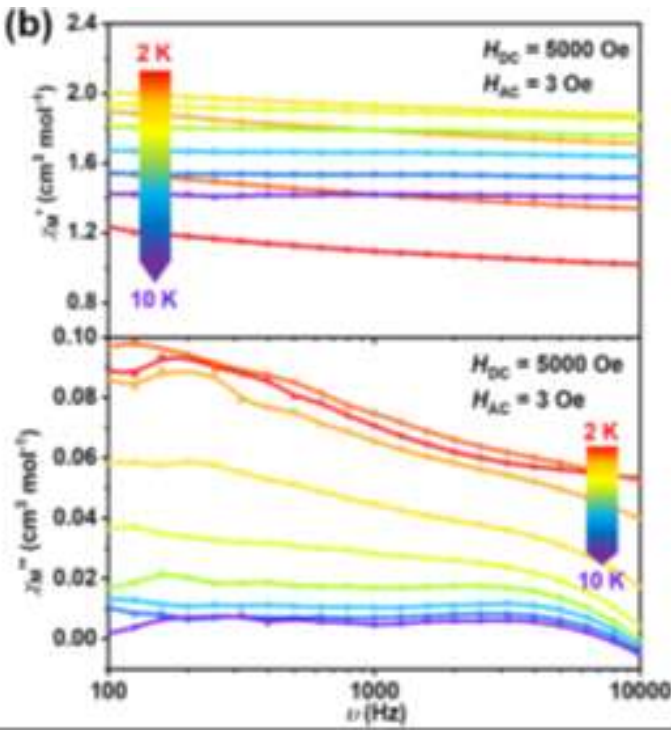

Figure S26. Temperature- (a) and frequency- (b) dependence of molar AC susceptibilities (upper: $\chi_{\mathrm{M}}{ }^{\prime}$; lower: $\chi_{\mathrm{M}}{ }^{\prime \prime}$ ) for $\mathbf{4 H o - L u t ~ u n d e r ~ a ~} 5000$ Oe of DC field and certain AC frequencies from 100 to $10000 \mathrm{~Hz}$.

\subsection{Magnetic dynamics of 1Er-THF}

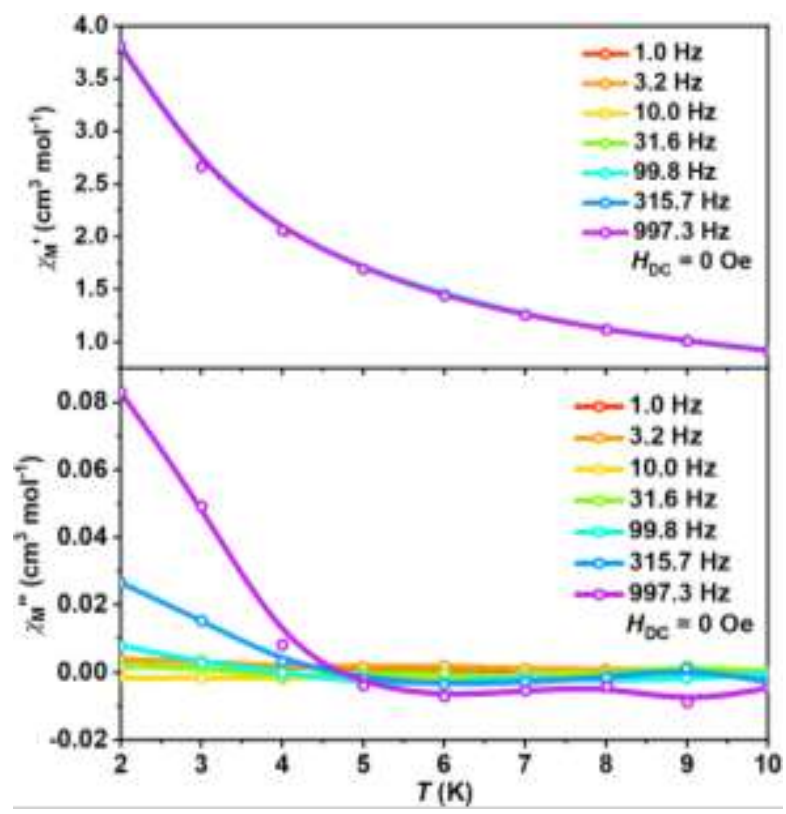

Figure S27. Temperature-dependence of molar AC susceptibilities (upper: $\chi_{\mathrm{M}}{ }^{\prime}$; lower: $\chi_{\mathrm{M}}{ }^{\prime \prime}$ ) for 1 Er-THF under a zero applied DC field and certain AC frequencies. 

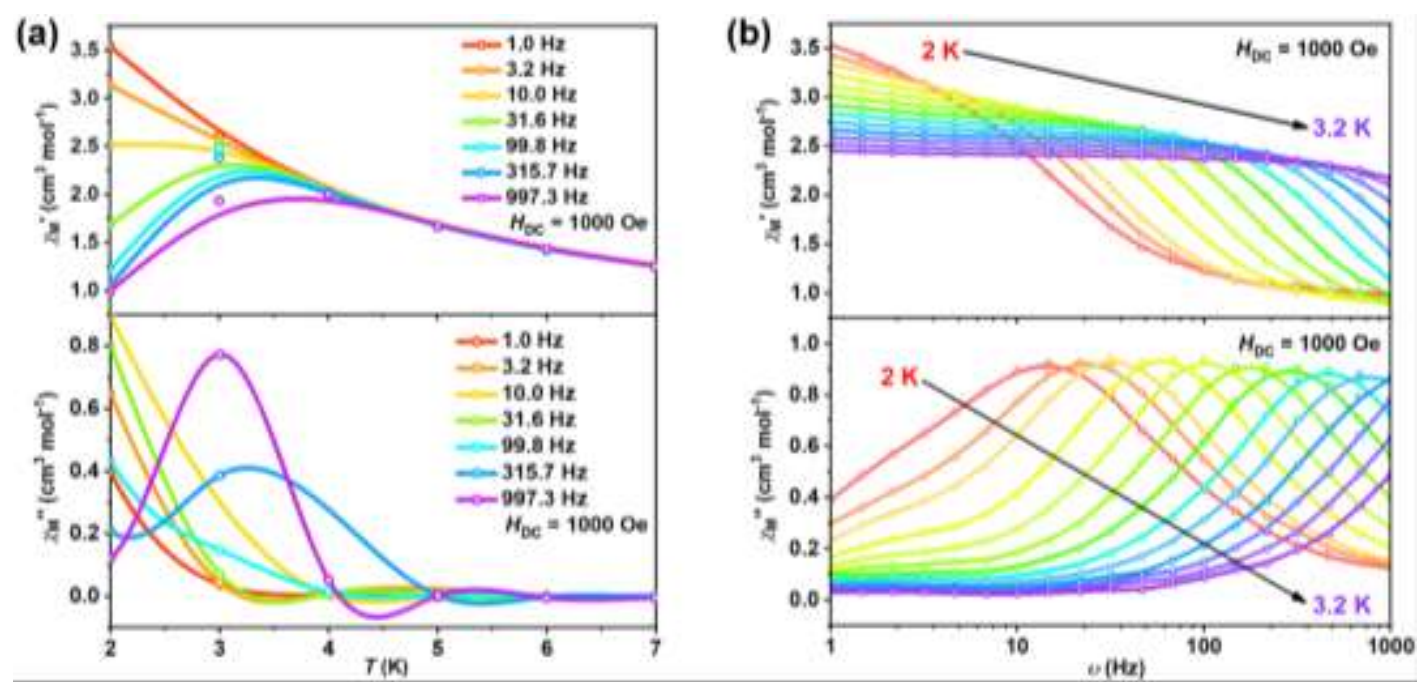

Figure S28. Temperature- (a) and frequency- (b) dependence of molar AC susceptibilities (upper: $\chi_{\mathrm{M}}{ }^{\prime}$; lower: $\chi_{\mathrm{M}}{ }^{\prime \prime}$ ) for 1Er-THF under a 1000 Oe applied DC field and certain AC frequencies from 1 to $1000 \mathrm{~Hz}$.
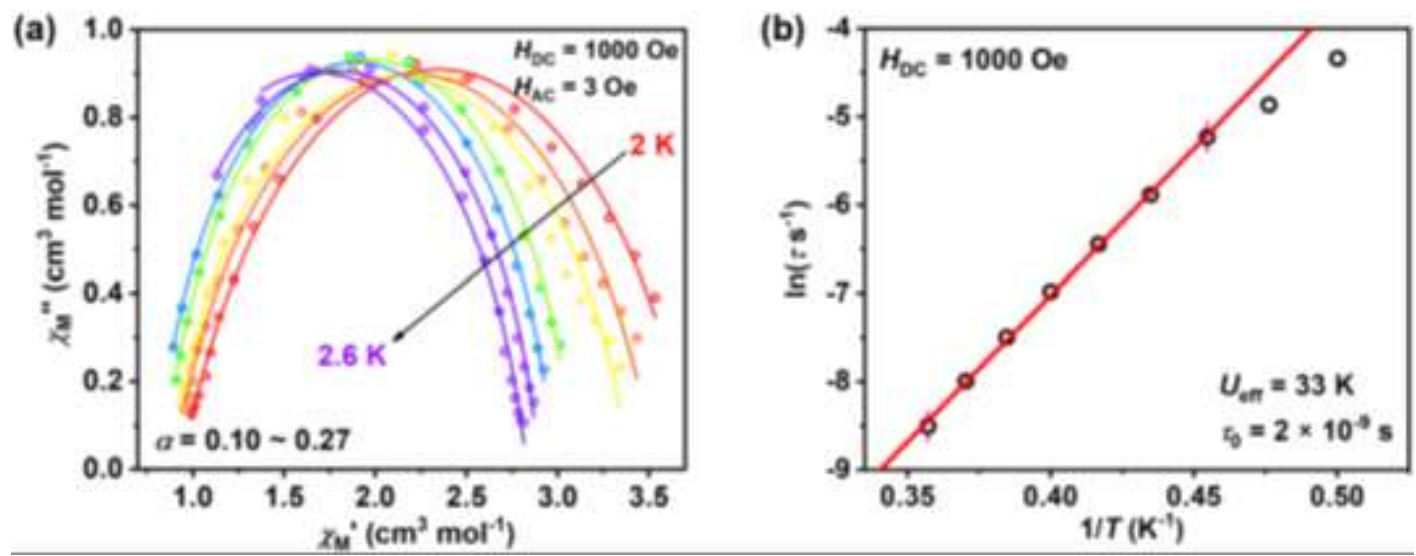

Figure S29. (a) Argand plots of $\chi_{\mathrm{M}}{ }^{\prime \prime}$ vs $\chi_{\mathrm{M}}{ }^{\prime}$ for 1 Er-THF under a 1000 Oe applied DC magnetic field. (b) Plot of natural $\log$ of relaxation time vs $1 / T$. The red solid line represents the best linear fitting using pure Orbach process $\tau^{-1}=\tau_{0}^{-}$ ${ }^{1} \exp \left(-\Delta / k_{\mathrm{B}} T\right)$. The fitting gives relaxation barrier $\Delta_{\mathrm{eff}} / k_{\mathrm{B}}=33 \mathrm{~K}$ and $\tau_{0}=2 \times 10^{-9} \mathrm{~s}$.

Table S10. Linear fitting parameters for the plot of $\ln \tau^{\sim} 1 / T$ using $\ln \tau=\Delta / k_{\mathrm{B}} T-\ln \tau_{0}$ under a 1000 Oe applied DC field for 1Er-THF.

\begin{tabular}{ccc}
\hline & Value & Standard Error \\
\hline $\ln \tau_{0}$ & -20.29602 & 0.1919 \\
$\Delta / k_{\mathrm{B}}$ & 33.19443 & 0.47512 \\
Adj. R-Square & 0.99877 & - \\
\hline
\end{tabular}


3.8. AC magnetic susceptibilities of $1 \mathrm{Tm}-\mathrm{THF}$
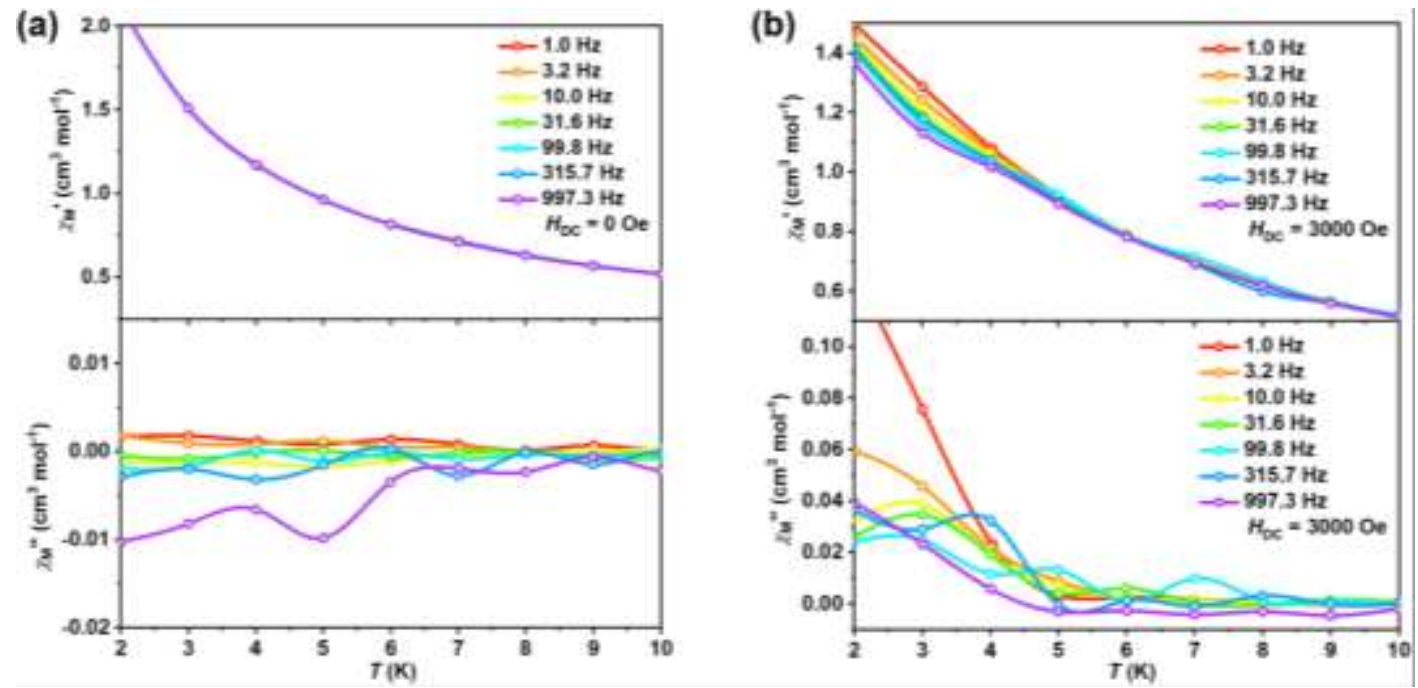

Figure S30. Temperature-dependence of molar AC susceptibilities (upper: $\chi_{\mathrm{M}}{ }^{\prime}$; lower: $\chi_{\mathrm{M}}{ }^{\prime \prime}$ ) for $\mathbf{1 T m}$-THF under a zero applied DC field (a) and a 3000 Oe DC field (b). 


\section{Computation}

Complete-active-space self-consistent field (CASSCF) calculations on the Tb" ${ }^{\prime \prime \prime}, \mathrm{Dy}{ }^{\prime \prime \prime}, \mathrm{Ho} \mathrm{O}^{\mathrm{II}}, \mathrm{Er} \mathrm{r}^{\mathrm{II}}$ and Tm'II (see Fig. S31. for the complete structure of complex 1Tb-THF; see Fig. S33. for the other four complexes) complexes on the basis of X-ray determined geometry have been carried out with MOLCAS 8.2 program package ${ }^{[5,6]}$.

For CASSCF calculations, the basis sets for all atoms are atomic natural orbitals from the MOLCAS ANO-RCC library: ANO-RCC-VTZP for Tb"', Dy"', Ho'"', Er"' and Tm"' ions; VTZ for coordinated C and $\mathrm{O}$; VDZ for distant atoms. The calculations employed the second order Douglas-Kroll-Hess Hamiltonian, where scalar relativistic contractions were taken into account in the basis set and the spin-orbit coupling was handled separately in the restricted active space state interaction (RASSISO) procedure. The active electrons in 7 active spaces include all $f$ electrons, CAS ( 8 in 7 for Tb"I', 9 in 7 for Dy'"', 10 in 7 for Ho'"I, 11 in 7 for Er ${ }^{\prime \prime \prime}$ and 12 in 7 for Tm ${ }^{\prime \prime \prime}$ ) in the CASSCF calculations. To exclude all the doubts, we calculated all the roots in the active space. We have mixed the maximum number of spin-free state which was possible with our hardware (all from 140 quintuplets and 68 from 500 triplets for Tb"'l; all from 21 sextets, 128 from 224 quadruplets and 130 from 490 doublets for Dy'l'; all from 35 quintuplets, 150 from 210 triplets and 120 from 196 singlets for Ho ${ }^{\text {III; }}$ all from 35 quadruplets and all from 112 doublets for Er'll; all from 21 triplets and all from 68 singlets for $\left.\mathrm{Tm}^{\prime \prime \prime}\right)$.

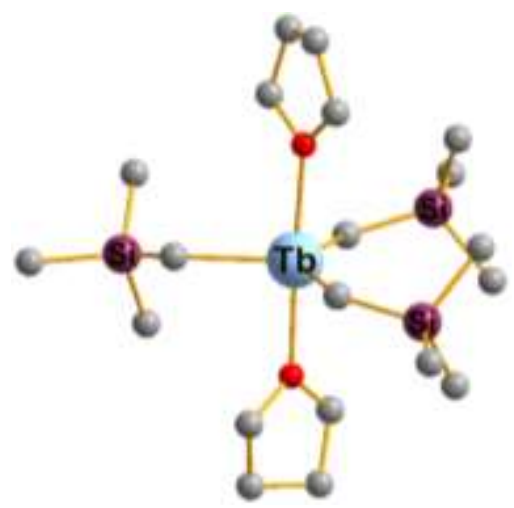

Figure S31. Calculated complete structure of complex 1Tb-THF; $\mathrm{H}$ atoms are omitted. 

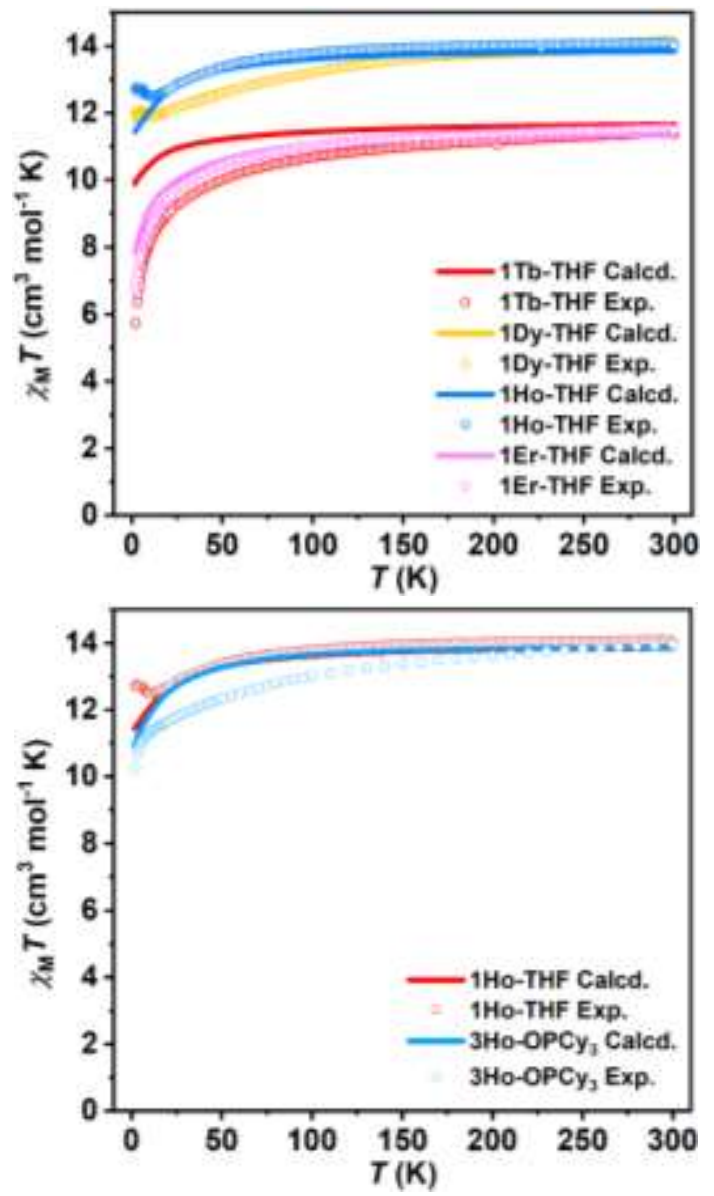

Figure S32. Comparisons of the calculated (solid line) and measured (circles) magnetic susceptibilities for $\mathbf{1}$ Ln-THF (Ln = Tb, Dy, Ho, Er) (top), and 3Ho-OPCy 3 (bottom).

Table S11. Calculated energy levels $\left(\mathrm{cm}^{-1}\right), \boldsymbol{g}\left(g_{\mathrm{x}}, g_{\mathrm{y}}, g_{\mathrm{z}}\right)$ tensors and $m_{\mathrm{J}}$ values of the lowest Kramers doublets for 1Dy-THF and 1Er-THF, non- Kramers doublets for 1Tb-THF, 1Ho-THF and 1Tm-THF.

\begin{tabular}{|c|c|c|c|c|c|c|c|c|}
\hline \multicolumn{5}{|c|}{ 1Tb-THF } & \multicolumn{4}{|c|}{ 1Dy-THF } \\
\hline & $E / \mathrm{cm}^{-1}$ & & $g$ & $m_{\jmath}$ & $E / \mathrm{cm}^{-1}$ & & $g$ & $m_{\jmath}$ \\
\hline \multirow{3}{*}{1} & \multirow{2}{*}{0.0} & $g_{\mathrm{x}}$ & 0.000 & \multirow{3}{*}{ \pm 6} & \multirow{3}{*}{0.0} & $g_{\mathrm{x}}$ & 0.093 & \multirow{3}{*}{ $\pm 15 / 2$} \\
\hline & & $g_{\mathrm{y}}$ & 0.000 & & & $g_{\mathrm{y}}$ & 0.205 & \\
\hline & 0.3 & $g_{\mathrm{z}}$ & 17.791 & & & $g_{z}$ & 19.405 & \\
\hline \multirow{3}{*}{2} & \multirow{2}{*}{38.3} & $g_{\mathrm{x}}$ & 0.000 & \multirow{3}{*}{ \pm 5} & \multirow{3}{*}{163.5} & $g_{\mathrm{x}}$ & 1.803 & \multirow{3}{*}{ $\pm 13 / 2$} \\
\hline & & $q_{y}$ & 0.000 & & & $q_{v}$ & 4.751 & \\
\hline & 41.7 & $g_{z}$ & 14.828 & & & $g_{z}$ & 13.169 & \\
\hline \multirow{3}{*}{3} & \multirow{2}{*}{110.2} & $g_{\mathrm{x}}$ & 0.000 & \multirow{3}{*}{ \pm 4} & \multirow{3}{*}{252.2} & $g_{\mathrm{x}}$ & 6.258 & \multirow{3}{*}{ $\pm 11 / 2$} \\
\hline & & $g_{\mathrm{y}}$ & 0.000 & & & $g_{\mathrm{y}}$ & 5.394 & \\
\hline & 121.3 & $g_{\mathrm{z}}$ & 11.032 & & & $g_{z}$ & 3.417 & \\
\hline \multirow[b]{2}{*}{4} & \multirow[b]{2}{*}{171.1} & & & \multirow[b]{2}{*}{0} & \multirow[b]{2}{*}{363.9} & $g_{\mathrm{x}}$ & 1.178 & \multirow[b]{2}{*}{ $\pm 9 / 2$} \\
\hline & & & & & & $g_{\mathrm{y}}$ & 2.687 & \\
\hline
\end{tabular}




\begin{tabular}{|c|c|c|c|c|c|c|c|c|}
\hline & & & & & & $g_{z}$ & 8.919 & \\
\hline \multirow{3}{*}{5} & \multirow{2}{*}{209.8} & $g_{\mathrm{x}}$ & 0.000 & \multirow{3}{*}{ \pm 1} & \multirow{3}{*}{480.0} & $g_{\mathrm{x}}$ & 0.836 & \multirow{3}{*}{ $\pm 3 / 2$} \\
\hline & & $g_{\mathrm{y}}$ & 0.000 & & & $g_{\mathrm{y}}$ & 1.002 & \\
\hline & 234.7 & $g_{z}$ & 14.985 & & & $g_{z}$ & 11.890 & \\
\hline \multirow{3}{*}{6} & \multirow{2}{*}{317.5} & $g_{\mathrm{x}}$ & 0.000 & \multirow{3}{*}{ \pm 2} & \multirow{3}{*}{559.9} & $g_{\mathrm{x}}$ & 0.075 & \multirow{3}{*}{ $\pm 7 / 2$} \\
\hline & & $g_{\mathrm{y}}$ & 0.000 & & & $g_{\mathrm{y}}$ & 0.098 & \\
\hline & 323.5 & $g_{z}$ & 14.508 & & & $g_{z}$ & 17.723 & \\
\hline \multirow{3}{*}{7} & \multirow{2}{*}{732.0} & $g_{\mathrm{x}}$ & 0.000 & \multirow{3}{*}{ \pm 3} & \multirow{3}{*}{576.3} & $g_{\mathrm{x}}$ & 0.027 & \multirow{3}{*}{ $\pm 1 / 2$} \\
\hline & & $g_{\mathrm{y}}$ & 0.000 & & & $g_{\mathrm{y}}$ & 0.065 & \\
\hline & 732.1 & $g_{z}$ & 17.885 & & & $g_{z}$ & 17.499 & \\
\hline \multirow{3}{*}{8} & & & & & \multirow{3}{*}{613.8} & $g_{\mathrm{x}}$ & 0.016 & \multirow{3}{*}{ $\pm 5 / 2$} \\
\hline & & & & & & $g_{\mathrm{y}}$ & 0.036 & \\
\hline & & & & & & $g_{z}$ & 18.207 & \\
\hline \multicolumn{5}{|c|}{ 1Ho-THF } & \multicolumn{4}{|c|}{ 1Er-THF } \\
\hline & $E / \mathrm{cm}^{-1}$ & & $g$ & $m_{\jmath}$ & $E / \mathrm{cm}^{-1}$ & & $g$ & $m_{\lrcorner}$ \\
\hline \multirow{3}{*}{1} & \multirow{2}{*}{0.0} & $g_{\mathrm{x}}$ & 0.000 & \multirow{3}{*}{ \pm 8} & \multirow{3}{*}{0.0} & $g_{\mathrm{x}}$ & 0.713 & \multirow{3}{*}{ $\pm 13 / 2$} \\
\hline & & $g_{\mathrm{y}}$ & 0.000 & & & $g_{\mathrm{y}}$ & 1.009 & \\
\hline & 0.05 & $g_{z}$ & 19.046 & & & $g_{z}$ & 15.515 & \\
\hline & 431 & $g_{\mathrm{x}}$ & 0.000 & & & $g_{\mathrm{x}}$ & 0.530 & \\
\hline 2 & & $g_{\mathrm{y}}$ & 0.000 & \pm 7 & 19.1 & $g_{\mathrm{y}}$ & 1.072 & $\pm 11 / 2$ \\
\hline & 44.1 & $g_{z}$ & 16.338 & & & $g_{\text {z }}$ & 13.102 & \\
\hline & 708 & $g_{\mathrm{x}}$ & 0.000 & & & $g_{\mathrm{x}}$ & 2.638 & \\
\hline 3 & & $g_{\mathrm{y}}$ & 0.000 & \pm 2 & 77.0 & $g_{\mathrm{y}}$ & 2.793 & $\pm 9 / 2$ \\
\hline & 14.1 & $g_{z}$ & 18.603 & & & $g_{\mathrm{z}}$ & 9.771 & \\
\hline & 124.9 & $g_{\mathrm{x}}$ & 0.000 & & & $g_{\mathrm{x}}$ & 0.003 & \\
\hline 4 & & $g_{\mathrm{y}}$ & 0.000 & \pm 6 & 118.8 & $g_{\mathrm{y}}$ & 0.133 & $\pm 15 / 2$ \\
\hline & 140.4 & $g_{z}$ & 12.509 & & & $g_{\mathrm{z}}$ & 17.454 & \\
\hline & & & & & & $g_{\mathrm{x}}$ & 4.373 & \\
\hline 5 & 163.0 & & & 0 & 135.9 & $g_{\mathrm{y}}$ & 5.012 & $\pm 7 / 2$ \\
\hline & & & & & & $g_{\mathrm{z}}$ & 9.146 & \\
\hline & 1719 & $g_{\mathrm{x}}$ & 0.000 & & & $g_{\mathrm{x}}$ & 0.127 & \\
\hline 6 & & $g_{\mathrm{y}}$ & 0.000 & \pm 3 & 210.0 & $g_{\mathrm{y}}$ & 1.690 & $\pm 3 / 2$ \\
\hline & $1 / 2.5$ & $g_{\mathrm{z}}$ & 19.320 & & & $g_{z}$ & 15.523 & \\
\hline 7 & 205.9 & $g_{\mathrm{x}}$ & 0.000 & \pm 1 & 239.6 & $g_{\mathrm{x}}$ & 0.677 & $\pm 1 / 2$ \\
\hline
\end{tabular}




\begin{tabular}{|c|c|c|c|c|c|c|c|c|}
\hline & \multirow{2}{*}{207.3} & $g_{\mathrm{v}}$ & 0.000 & & & $g_{\mathrm{y}}$ & 1.453 & \\
\hline & & $g_{2}$ & 12.034 & & & $g_{z}$ & 14.013 & \\
\hline \multirow{3}{*}{8} & 255.1 & $g_{\mathrm{x}}$ & 0.000 & \multirow{3}{*}{ \pm 4} & \multirow{3}{*}{290.1} & $g_{\mathrm{x}}$ & 0.043 & \\
\hline & \multirow{2}{*}{258.7} & $g_{\mathrm{y}}$ & 0.000 & & & $g_{\mathrm{y}}$ & 0.095 & $\pm 5 / 2$ \\
\hline & & $g_{2}$ & 15.130 & & & $g_{z}$ & 17.143 & \\
\hline \multirow{3}{*}{9} & \multirow{2}{*}{269.3} & $g_{\mathrm{x}}$ & 0.000 & \multirow{3}{*}{ \pm 5} & & & & \\
\hline & & $g_{\mathrm{v}}$ & 0.000 & & & & & \\
\hline & 270.2 & $g_{2}$ & 17.399 & & & & & \\
\hline & & $1 T \mathrm{~m}$ & & & & & & \\
\hline & $E / \mathrm{cm}^{-1}$ & $g$ & $m_{J}$ & $\mathrm{E} / \mathrm{cm}^{-1}$ & & & & \\
\hline \multirow{3}{*}{1} & 0.0 & $g_{\mathrm{x}}$ & 0.000 & \multirow{3}{*}{ \pm 6} & & & & \\
\hline & \multirow{2}{*}{0.3} & $g_{\mathrm{v}}$ & 0.000 & & & & & \\
\hline & & $g_{2}$ & 13.824 & & & & & \\
\hline \multirow{3}{*}{2} & \multirow{2}{*}{35.3} & $g_{\mathrm{x}}$ & 0.000 & \multirow{3}{*}{ \pm 5} & & & & \\
\hline & & $g_{\mathrm{v}}$ & 0.000 & & & & & \\
\hline & 36.4 & $g_{2}$ & 11.980 & & & & & \\
\hline \multirow{3}{*}{3} & \multirow{2}{*}{107.6} & $g_{\mathrm{x}}$ & 0.000 & \multirow{3}{*}{ \pm 1} & & & & \\
\hline & & $g_{\mathrm{v}}$ & 0.000 & & & & & \\
\hline & 122.5 & $g_{2}$ & 9.262 & & & & & \\
\hline \multirow{3}{*}{4} & \multirow{2}{*}{223.3} & $g_{\mathrm{x}}$ & 0.000 & \multirow{3}{*}{ \pm 3} & & & & \\
\hline & & $g_{\mathrm{y}}$ & 0.000 & & & & & \\
\hline & 273.1 & $g_{2}$ & 6.828 & & & & & \\
\hline 5 & 372.3 & & & 0 & & & & \\
\hline & 396.6 & $g_{\mathrm{x}}$ & 0.000 & & & & & \\
\hline 6 & & $g_{\mathrm{y}}$ & 0.000 & \pm 2 & & & & \\
\hline & 450.9 & $g_{z}$ & 2.519 & & & & & \\
\hline & 5859 & $g_{\mathrm{x}}$ & 0.000 & & & & & \\
\hline 7 & & $g_{\mathrm{v}}$ & 0.000 & \pm 4 & & & & \\
\hline & 592.1 & $g_{z}$ & 0.048 & & & & & \\
\hline
\end{tabular}



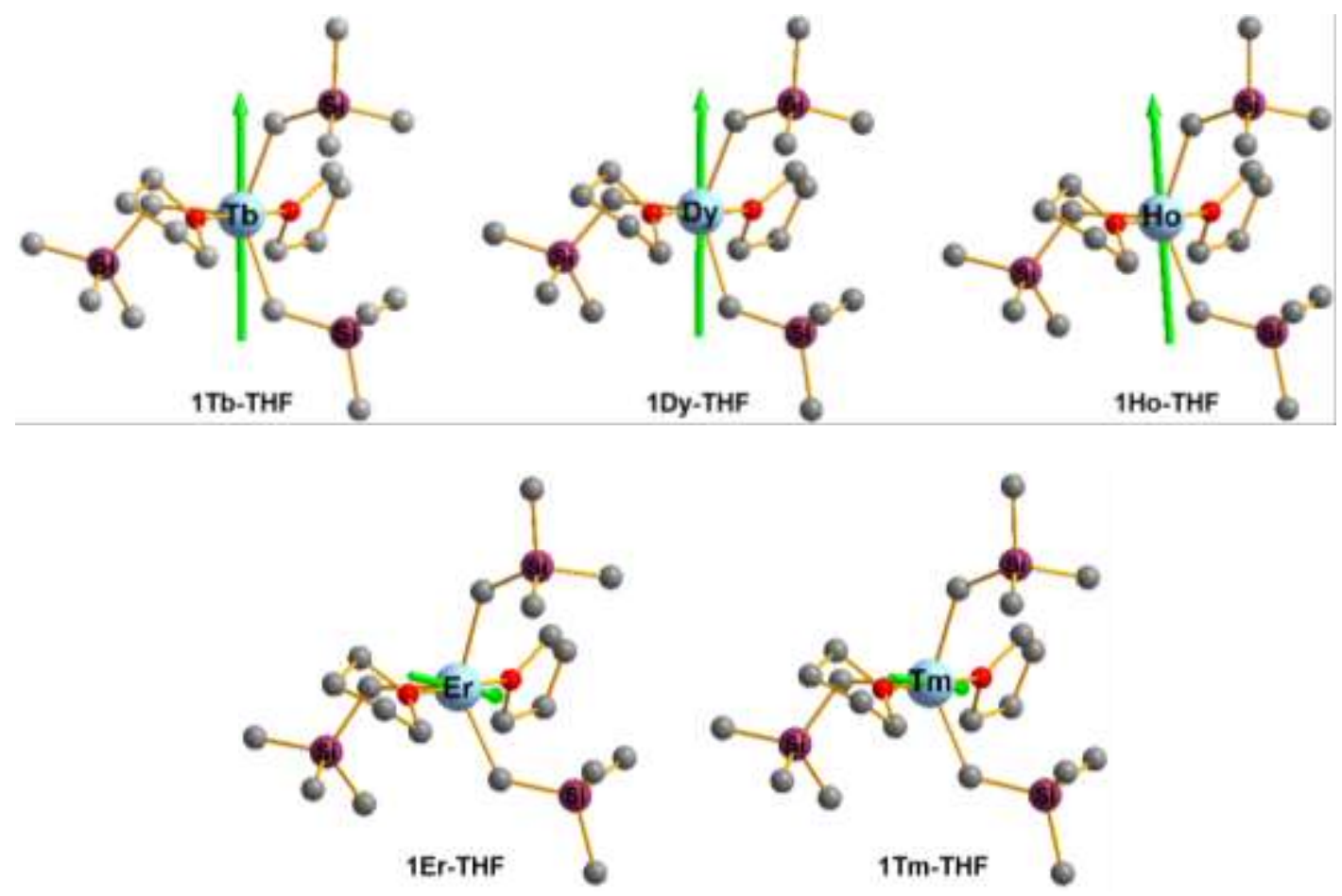

Figure S33. Orientations of the local main magnetic axes (green arrows) of the ground Kramers doublets for 1DyTHF and 1Er-THF, non- Kramers doublets for 1Tb-THF, 1Ho-THF and 1Tm-THF.

Table S12. The calculated anisotropic $g$ tensors at ground states for 1Ho-THF, 2Ho-QN and 3Ho-OPCy.

\begin{tabular}{c|c|ccc}
\hline State & $g$ & 1Ho-THF & 2Ho-QN & 3Ho-OPC $_{\mathbf{3}}$ \\
\hline \multirow{4}{*}{ Ground State } & $g_{x}$ & 0 & $0 / 0$ & 0 \\
& $g_{y}$ & 0 & $0 / 0$ & 0 \\
& $g_{z}$ & 19.0488 & $4.0157 / 4.0178$ & 18.7603 \\
\hline \multirow{3}{*}{$\mathbf{1}^{\text {st }}$ Excited State } & $g_{x}$ & 0 & $0 / 0$ & 0 \\
& $g_{y}$ & 0 & $0 / 0$ & 0 \\
& $g_{z}$ & 16.3370 & $0.1520 / 0.0289$ & 18.3227 \\
\hline \multirow{3}{*}{$\mathbf{2}^{\text {nd }}$ Excited State } & $g_{x}$ & 0 & 0 & 0 \\
& $g_{y}$ & 0 & 0 & 15.4791 \\
\hline
\end{tabular}




\section{References}

[1] (a) H. Schumann, D. M. M. Freckmann and S. Dechert, Z. Anorg. Allg. Chem. 2002, 628, 2422. (b) H. Schumann, J. Müller, J. Organomet. Chem. 1978, 146, C5.

[2] O. V. Dolomanov, L. J. Bourhis, R. J. Gildea, J. A. K. Howard and H. Puschmann, J. Appl. Cryst. 2009, 42, 339.

[3] L. Palatinus and G. Chapuis, J. Appl. Cryst. 2007, 40, 786.

[4] G. A. Bain and J. F. Berry, J. Chem. Edu. 2008, 85, 532.

[5] G. Karlström, R. Lindh, P.-Å. Malmqvist, B. O. Roos, U. Ryde, V. Veryazov, P.-O. Widmark, M. Cossi, B. Schimmelpfennig, P. Neogrady, L. Seijo, MOLCAS: a Program Package for Computational Chemistry. Comput. Mater. Sci. 2003, 28, 222.

[6] M. J. Frisch, G. W. Trucks, H. B. Schlegel, G. E. Scuseria, M. A. Robb, J. R. Cheeseman, G. Scalmani, V.Barone, B. Mennucci, G. A. Petersson, H. Nakatsuji, M. Caricato, X. Li, H. P. Hratchian, A. F. Izmaylov, J. Bloino,G. Zheng, J. L. Sonnenberg, M. Hada, M. Ehara, K. Toyota, R. Fukuda, J. Hasegawa, M. Ishida, T. Nakajima, Y. Honda, O. Kitao, H. Nakai, T. Vreven, J. A. Montgomery, Jr., J. E. Peralta, F. Ogliaro, M. Bearpark, J. J. Heyd, E. Brothers, K. N. Kudin, V. N. Staroverov, T. Keith, R. Kobayashi, J. Normand, K. Raghavachari, A. Rendell, J. C. Burant, S. S. lyengar, J. Tomasi, M. Cossi, N. Rega, J. M. Millam, M. Klene, J. E. Knox, J. B. Cross, V. Bakken, C. Adamo, J. Jaramillo, R. Gomperts, R. E. Stratmann, O. Yazyev, A. J. Austin, R. Cammi, C. Pomelli, J. W. Ochterski, R. L. Martin, K. Morokuma, V. G. Zakrzewski, G. A. Voth, P. Salvador, J. J. Dannenberg, S. Dapprich, A. D. Daniels, O. Farkas, J. B. Foresman, J. V. Ortiz, J. Cioslowski and D. J. Fox, Revision D.01 ed., Gaussian, Inc., Wallingford CT, 2013. 